\title{
TU/e EnNHONEN

\section{Asymptotic solution and numerical simulation of homogeneous condensation in expansion cloud chambers}

\section{Citation for published version (APA):}

Delale, C. F., Muitjens, M. J. E. H., \& Dongen, van, M. E. H. (1996). Asymptotic solution and numerical simulation of homogeneous condensation in expansion cloud chambers. Journal of Chemical Physics, 105(19), 8804-8821. https://doi.org/10.1063/1.472631

DOI:

10.1063/1.472631

Document status and date:

Published: 01/01/1996

\section{Document Version:}

Publisher's PDF, also known as Version of Record (includes final page, issue and volume numbers)

\section{Please check the document version of this publication:}

- A submitted manuscript is the version of the article upon submission and before peer-review. There can be important differences between the submitted version and the official published version of record. People interested in the research are advised to contact the author for the final version of the publication, or visit the $\mathrm{DOI}$ to the publisher's website.

- The final author version and the galley proof are versions of the publication after peer review.

- The final published version features the final layout of the paper including the volume, issue and page numbers.

Link to publication

\section{General rights}

Copyright and moral rights for the publications made accessible in the public portal are retained by the authors and/or other copyright owners and it is a condition of accessing publications that users recognise and abide by the legal requirements associated with these rights.

- Users may download and print one copy of any publication from the public portal for the purpose of private study or research.

- You may not further distribute the material or use it for any profit-making activity or commercial gain

- You may freely distribute the URL identifying the publication in the public portal.

If the publication is distributed under the terms of Article 25fa of the Dutch Copyright Act, indicated by the "Taverne" license above, please follow below link for the End User Agreement:

www.tue.nl/taverne

Take down policy

If you believe that this document breaches copyright please contact us at:

openaccess@tue.nl

providing details and we will investigate your claim. 


\title{
Asymptotic solution and numerical simulation of homogeneous condensation in expansion cloud chambers
}

\author{
C. F. Delale, ${ }^{\text {a) }}$ M. J. E. H. Muitjens, and M. E. H. van Dongen \\ Department of Physics, Eindhoven University of Technology, 5600 MB Eindhoven, The Netherlands
}

(Received 15 April 1996; accepted 9 August 1996)

\begin{abstract}
Asymptotic solution of homogeneous condensation in expansion cloud chambers in different droplet growth regimes is presented. In particular an exactly solvable droplet growth model ranging between the Hertz-Knudsen and continuum droplet growth laws is introduced. The distinct condensation zones in each droplet growth regime are identified by the asymptotic solution of the condensation rate equation and the results are compared with those of direct numerical simulations using the classical nucleation theory. Excellent qualitative agreement is reached despite some minor quantitative differences in some of the condensation zones arising from the nature of the asymptotic solution in these zones. (C) 1996 American Institute of Physics. [S0021-9606(96)52142-2]
\end{abstract}

\section{INTRODUCTION}

Homogeneous condensation is the process of generation of clusters of vapor molecules which act as condensation nuclei by thermal fluctuations (homogeneous nucleation) followed by the growth of these nuclei into droplets (droplet growth). This nonequilibrium process appears in special environments in physics, chemistry, biology, and many areas of engineering. It entered atmospheric physics as early as 1880 by Aitken's investigations ${ }^{1}$ and with the invention of the supersonic nozzle by de Laval, it led to investigations in conjunction with steam turbines. ${ }^{2}$ Wilson ${ }^{3}$ examined homogeneous condensation in his invention of the classical cloud chamber which later was used for different applications in physics. The theory was first formulated using homogeneous nucleation and droplet growth theories combined with the equations of motion by Oswatitsch. ${ }^{4}$ Detailed experimental and theoretical investigations ${ }^{5-12}$ in different configurations have been performed since then. Complete analytical solutions to the theory of homogeneous condensation in supersonic nozzles have been possible in the last two decades by the pioneering work of Blythe and Shih ${ }^{13}$ and Clarke and Delale ${ }^{14}$ using an asymptotic method. The method has been refined $^{15}$ and extended to the investigation of homogeneous condensation in shock tubes. ${ }^{16}$

The aim of the present paper is to apply the asymptotic method for the investigation of homogeneous condensation in expansion cloud chambers. For this reason the detailed physical mechanism of homogeneous condensation (homogeneous nucleation and droplet growth) is discussed in Sec. II. The homogeneous nucleation equation is cast into a normalized functional form independent of the particular theory to be employed. The growth mechanism is given a general consideration to cover growth regimes from free molecular (Hertz-Knudsen) to continuum. In particular an exactly

\footnotetext{
${ }^{a}$ Permanent address: Department of Mechanical Engineering, Istanbul University, Avcilar 34850, Istanbul and Department of Mathematics, TÜBITAK Marmara Research Center, 41470 Gebze, Kocaeli, Turkey.
}

solvable droplet growth model in the transition regime which yields growth rates ranging from Hertz-Knudsen to continuum growth rates is constructed for an analytical solution and is compared with the most widely used transition growth expressions. The condensation rate equation for the molar fraction is then constructed from the nucleation and growth equations for different droplet growth regimes and is solved in conjunction with the governing equations of expansion cloud chambers by an asymptotic method in the limit of relatively slow nucleation process followed by rapid droplet growth. The distinct condensation zones with complete analytical structure are then identified by the asymptotic solution. Finally the results for different initial mixture pressures (corresponding to different droplet growth regimes) are compared with direct numerical simulations of the rate equation combined with the equations of motion. Excellent qualitative and reasonable quantitative agreement between the asymptotic solution and numerical simulations of the rate equation is achieved despite some quantitative differences which mainly arise from the asymptotic nature of the solution.

\section{THEORY OF HOMOGENEOUS CONDENSATION IN EXPANSION CLOUD CHAMBERS}

We consider a mixture of a condensable vapor and a carrier gas in an expansion chamber with initial total pressure $p_{0}^{\prime}$, initial partial vapor pressure $\left(p_{v}^{\prime}\right)_{0}$ and initial temperature $T_{0}^{\prime}$. We assume that the mixture contains no impurities such as dust particles, ions, etc. so that homogeneous nucleation sets in during the expansion of the mixture in metastable states. As the expansion valve is opened at the initial time $t_{0}^{\prime}=0$, the mixture expands isentropically for a while until the vapor becomes saturated with no detectable condensation. As the mixture continues to expand almost isentropically further on in metastable states of the vapor, homogeneous nucleation sets in resulting in the formation of condensation nuclei which rapidly grow into droplets. When the number of growing droplets reach a certain value, dropwise condensation becomes detectable (onset of condensa- 
tion). Consequently significant deviations in the total pressure and temperature of the mixture from their isentropic values become observable, the nucleation rate reaches its peak value with a subsequent decay, and vapor depletion due to droplet growth becomes noticable. The two-phase mixture then relaxes toward saturated equilibrium states due to further growth of the droplets. The objective is to locate the onset of condensation, to predict the shape of the nucleation curve in time and to obtain the structure of the relaxation zone over a wide range of initial pressures ranging from low to relatively high values.

\section{A. Governing equations}

We herein present the governing equations of expansion cloud chambers in the two-phase homogeneous dispersed droplet regime assuming no slip between droplets and the surrounding mixture. We further assume that the droplets and the surrounding mixture are at the same temperature $T^{\prime}$ so that the heat released per mole of the mixture is equal to $y L^{\prime}\left(T^{\prime}\right)$ where $y$ is the molar fraction of the condensate and $L^{\prime}\left(T^{\prime}\right)$ is the molar latent heat of condensation corresponding to temperature $T^{\prime}$ (one-temperature model). The total mixture pressure $p^{\prime}$ can either be precisely measured or it can be related to the volume change during the expansion. This helps us to bypass any consideration of the momentum equation. The conservation of energy then leads to the equation

$$
n^{\prime} \frac{d h^{\prime}}{d t^{\prime}}=\frac{d p^{\prime}}{d t^{\prime}}
$$

where $t^{\prime}$ is the time, $n^{\prime}$ is the molar density of the mixture, and $h^{\prime}$ is the mixture molar enthalpy defined by

$$
h^{\prime}=c_{p m}^{\prime} T^{\prime}-y L^{\prime}\left(T^{\prime}\right)+\text { constant. }
$$

In Eq. (2) $c_{p m}^{\prime}$ denotes the molar specific heat of the mixture at constant pressure and is related to the molar specific heats at constant pressure of the carrier gas $c_{p i}^{\prime}$ and of the vapor $c_{p v}^{\prime}$ by

$$
c_{p m}^{\prime}=\left(1-y_{v 0}\right) c_{p i}^{\prime}+y_{v 0} c_{p v}^{\prime},
$$

where $y_{v 0}$ is the initial molar fraction of the condensable vapor. On the other hand the thermal equation of state of the mixture is constructed by Dalton's law

$$
p^{\prime}=p_{i}^{\prime}+p_{v}^{\prime}
$$

where $p_{i}^{\prime}$ and $p_{v}^{\prime}$, respectively, denote the partial vapor pressure of the carrier gas and of the vapor. Assuming that the condensable vapor and the carrier gas both obey the ideal gas law, we obtain the thermal equation of state of the mixture as

$$
p^{\prime}=(1-y) n^{\prime} T^{\prime} \text {. }
$$

We now normalize the flow variables with respect to their saturation values during expansion, namely

$$
p \equiv \frac{p^{\prime}}{p_{s}^{\prime}}, \quad T \equiv \frac{T^{\prime}}{T_{s}^{\prime}}, \text { and } n \equiv \frac{n^{\prime}}{n_{s}^{\prime}},
$$

where we refer to saturation values by subscript $s$ (all primed variables denote actual quantities whereas the unprimed variables correspond to their normalized values). Furthermore we normalize the molar specific enthalpy $h^{\prime}$, the molar specific heat of the mixture at constant pressure $c_{p m}^{\prime}$, and the molar latent heat $L^{\prime}\left(T^{\prime}\right)$ by

$$
h \equiv \frac{h^{\prime}}{\mathscr{B} T_{s}^{\prime}}, \quad c_{p m} \equiv \frac{c_{p m}^{\prime}}{\mathscr{R}}, \text { and } L(T) \equiv \frac{L^{\prime}\left(T^{\prime}\right)}{\mathscr{R} T_{s}^{\prime}},
$$

where $\mathscr{B}$ is the universal gas constant. On the other hand the time $t^{\prime}$ is normalized as

$$
t \equiv \frac{t^{\prime}}{t_{r}^{\prime}}
$$

where $t_{r}^{\prime}$ is the expansion time given by

$$
\frac{1}{t_{r}^{\prime}} \equiv-\left(\frac{1}{p^{\prime}} \frac{d p^{\prime}}{d t^{\prime}}\right)_{t_{0}^{\prime}=0} \text {. }
$$

For most applications in the range of temperatures considered, the normalized molar latent heat can be taken as a linear function in $T$ as

$$
L(T) \equiv a T+b .
$$

With the above normalization Eqs. (1), (2), (5), and (10) can be solved for $T$ and $n$ to yield

$$
\begin{aligned}
T(t)= & \frac{[p(t)]^{1 / c_{p m}}}{1-\frac{b}{c_{p m}} y(t)}+\frac{a y(t)}{c_{p m}-b y(t)} \\
& +\frac{a[p(t)]^{1 / c_{p m}}}{c_{p m}\left[c_{p m}-b y(t)\right]} \phi(t), \\
n(t)= & \frac{p(t)}{[1-y(t)] T(t)},
\end{aligned}
$$

where

$$
\phi(t) \equiv \int_{t_{s}}^{t} y(\xi)[p(\xi)]^{-\left(1+c_{p m}\right) / c_{p m}} d \xi .
$$

In particular Eqs. (11) and (12) reduce to the classical isentropic relations for noncondensing flows $(y \equiv 0)$. For condensing flows Eqs. (11) and (12) yield the solution for the temperature $T$ and molar density $n$ of the mixture at any given instant $t$ in terms of the total pressure $p$ and the molar fraction of the condensate $y$. In what follows the pressure will be assumed to be given as a function of time $[p=p(t)]$ either by direct measurements or by assigning a prescribed form, and the condensate molar fraction $y$ will be obtained from the asymptotic solution of the condensation rate equation.

\section{B. Homogeneous nucleation and droplet growth}

The condensation of vapor in the supersaturated state occurs by homogeneous nucleation followed by rapid growth. Homogeneous nucleation is the process of formation of clusters of critical size in metastable equilibrium with the 
surrounding vapor in the absence of any foreign particles. The most well-known and widely used theory of homogeneous nucleation is the classical nucleation theory of Becker, Döring, and Zeldovich, ${ }^{17,18}$ which is based on the capillarity approximation. The more systematic statistical mechanical theories of Lothe and Pound ${ }^{19}$ and of Reiss, Katz, and Cohen $^{20}$ have helped improving our understanding of the phenomenon. Unfortunately none of these theories have been successful in comparison with measured nucleation rates for most substances over a wide temperature range (some have been successful for particular substances over restricted temperature ranges while they have proved to be considerably off in comparison with measured nucleation rates for the remaining substances). Recent advances in nucleation theory has mostly been in the direction of constructing semiphenomenological models based on Fisher's droplet theory of condensation. ${ }^{21}$ Among these models the ones proposed by Dillmann and Meier, ${ }^{22}$ Delale and Meier, ${ }^{23}$ and Kalikmanov and van Dongen ${ }^{24}$ seem to yield better agreement than the classical theory in comparison with experiments for a variety of substances over a wide range of temperatures. All of the above theories can nevertheless be cast into a unique functional form for the normalized steady-state nucleation rate $J$ as

$$
J \equiv \frac{J^{\prime}}{\zeta^{\prime}}=\Sigma(p, T, y) \exp \left[-K^{-1} B(p, T, y)\right],
$$

where $J^{\prime}$ is the actual nucleation rate (number of clusters of critical size formed per unit volume per unit time), $\zeta^{\prime}$ is a normalization constant for nucleation, $B$ is the normalized activation function, $K$ is the nucleation parameter, much less than unity, and $\Sigma$ is the normalized pre-exponential factor of the order of unity. For applications the parameters $K$ and $\zeta^{\prime}$, and the functions $B$ and $\Sigma$ are identified from the particular homogeneous nucleation theory to be employed. For our consideration of homogeneous condensation in expansion cloud chambers, the functional form (14) can be employed, without loss of generality, to characterize the number of critical clusters that grow into droplets in the local thermodynamic state $(p, T, y)$.

The growth of droplets is governed by interfacial rates of heat, mass and momentum transfer. Taking into account the no slip condition between droplets and the surrounding mixture and the condition that the droplets are at the same temperature of the mixture in the two-phase dispersed droplet model considered herein, the droplet growth law can be expressed by a single mass transfer equation to a spherical droplet. This mass transfer equation can be written in terms of a single nondimensional number, the Nusselt number $\mathrm{Nu}$, as

$$
\mathrm{Nu}=\frac{\dot{M}^{\prime} p^{\prime}}{2 \pi r^{\prime} \Delta^{\prime}\left(p_{v}^{\prime}-p_{r}^{\prime}\right)},
$$

where $\dot{M}^{\prime}$ is the rate of mass transfer to a spherical droplet of radius $r^{\prime}$ and is defined by

$$
\dot{M}^{\prime} \equiv 4 \pi r^{\prime 2} \rho_{l}^{\prime} \frac{d r^{\prime}}{d t^{\prime}}
$$

with $\rho_{l}^{\prime}$ denoting the mass density of the condensed phase, $p_{r}^{\prime}$ is the vapor pressure of the droplet surface and is related to the saturation pressure of planar interphase $p_{v s, \infty}^{\prime}$ at $T^{\prime}$ by the well-known Gibbs-Thomson relation

$$
p_{r}^{\prime}=p_{v s, \infty}^{\prime}\left(T^{\prime}\right) \exp \left(\frac{2 \sigma^{\prime}}{r^{\prime}} \frac{v^{\prime}}{k T^{\prime}}\right) .
$$

In Eq. (17) $\sigma^{\prime}$ denotes the surface tension, $k$ is the Boltzmann constant, $v^{\prime}$ is the molecular volume, and $\Delta^{\prime}$ is a modified diffusion coefficient defined by

$$
\Delta^{\prime} \equiv \frac{D^{\prime} \rho^{\prime} R_{m}}{R_{v}\left(1-p_{v}^{\prime} / p^{\prime}\right)}
$$

with $D^{\prime}$ denoting the diffusion coefficient, $R_{m}$ the mixture gas constant, and $R_{v}$ the vapor gas constant. Equations (15) and (16) yield the appropriate droplet growth law for the no-slip model assumed provided that an expression for the Nusselt number $\mathrm{Nu}$ (or equivalently the Sherwood number $\mathrm{Sh}$ ) can be found. It can be shown by dimensional analysis that for the no-slip two-phase model considered the Nusselt number $\mathrm{Nu}$ for mass transfer depends on the following nondimensional group as:

$$
\mathrm{Nu}=f\left(\mathrm{Kn}, \mathrm{Pr}, \mathrm{Sc}, \mathrm{Fo}, g_{\infty}\right) .
$$

In Eq. (19), $\mathrm{Kn}$ is the Knudsen number defined by

$$
\mathrm{Kn} \equiv \frac{l^{\prime}}{2 r^{\prime}}
$$

with $l^{\prime}$ denoting the mean free path of molecules in the gas mixture far from the droplet. $\mathrm{Pr}$ is the Prandtl number defined by

$$
\operatorname{Pr} \equiv \frac{c_{p m}^{\prime} \eta^{\prime}}{k^{\prime}}
$$

with $c_{p m}^{\prime}$ denoting the specific heat at constant pressure of the gas mixture, $k^{\prime}$ the thermal conductivity, and $\eta^{\prime}$ the viscosity of the gas mixture. Sc is the Schmidt number defined by

$$
\mathrm{Sc} \equiv \frac{\eta^{\prime}}{\rho^{\prime} D^{\prime}},
$$

which is the ratio of momentum diffusivity to mass diffusivity. Fo is the Fourier number defined by

$$
\mathrm{FO} \equiv \frac{a^{\prime} t_{r}^{\prime}}{r^{\prime 2}},
$$

which is a nondimensional group for the unsteadiness with $a^{\prime}$ denoting the thermal diffusivity of the mixture, and $g_{\infty}$ refers to a group of parameters characterizing ratios of properties in the gas mixture such as the dilution ratio $p_{v}^{\prime} / p^{\prime}$ or the ratio of gas constants $R_{m} / R_{v}$. The Fourier number Fo, which characterizes the influence of nonstationary change of state on the droplet growth rate, assumes very high values and remains almost constant during expansions in most applications; therefore, it need not be taken into account for most applications (the quasisteady approximation). The groups Pr and Sc are properties of the gas mixture only and 
they strictly depend on the dilution ratio $p_{v}^{\prime} / p^{\prime}$. For most cases their values are both close to unity and can only weakly influence the form (19) for the Nussult number. The Knudsen number $\mathrm{Kn}$, on the other hand, is substantially determined by pressure and droplet size and for the no-slip model assumed, it essentially determines the form (19) of the droplet growth law. If $\mathrm{Kn} \ll 1$, the gas mixture acts as a continuum. The problem of determining the Nusselt number for the no-slip model assumed then reduces to the steady-state diffusion problem and Eq. (19) can then be written as

$$
\mathrm{Nu}_{C}=2 \text {. }
$$

Equations (15), (16), (17), and (24) then determine the continuum droplet growth law for the two-phase model assumed (the droplets and gas mixture are at the same temperature, there is no velocity slip between the carrier gas and the dispersed droplets, etc.). If $\mathrm{Kn} \gg 1$ (the free molecular regime), heat conduction, viscous shear, and diffusion, being properties of the continuum, lose their physical relevance. The transfer rates of heat and momentum should then be expressed as the sum of net energy or momentum transferred individually by the molecules colliding with the droplet. The mass transfer rate can similarly be obtained by the net mass of vapor condensing on the droplet and, for the model considered, can be calculated by (for details see Gyarmathy ${ }^{25}$ )

$$
\dot{M}^{\prime}=4 \pi r^{\prime 2} \alpha_{M}^{\prime} \frac{p^{\prime}}{\sqrt{2 \pi R_{v} T^{\prime}}}\left(p_{v}^{\prime}-p_{r}^{\prime}\right)
$$

with $\alpha_{M}^{\prime}$ denoting the mass accommodation coefficient, which is the ratio of sticking molecules to those impinging on it and is to be determined experimentally. Equations (16), (17), and (25) then determine the free molecular or HertzKnudsen growth law. In particular the continuum and free molecular growth rates can each be cast into a single equation if we define the normalized droplet radius $r$ by

$$
r \equiv \frac{r^{\prime}}{r_{d}^{\prime}} .
$$

In Eq. (26) $r_{d}^{\prime}$ is a conveniently defined normalization constant for droplet growth given by

$$
r_{d}^{\prime} \equiv\left(\frac{3 n_{s}^{\prime}}{4 \pi \zeta^{\prime} \overrightarrow{n_{l}} t_{r}^{\prime}}\right)^{1 / 3}
$$

with $\overrightarrow{n_{l}}$ denoting the actual molar density of the condensed phase. We will herein neglect the so-called Kelvin effect, i.e., we take $p_{r}^{\prime}=p_{v s, \infty}^{\prime}$ in Eq. (17). Utilizing Eqs. (15), (16), and (24) together with the condition $p_{r}^{\prime}=p_{v s, \infty}^{\prime}$, the continuum droplet growth law can be written in normalized form as

$$
\frac{d r}{d t}=\frac{1}{r} \lambda_{C} \Omega_{C}(p, T, y) .
$$

Here $\lambda_{C}$ will be referred to as the continuum growth parameter and $\Omega_{C}$, which depends on the local thermodynamic state, will be referred to as the continuum growth function.
Similarly utilizing Eqs. (16) and (25) and neglecting the Kelvin effect, we obtain the free molecular or HertzKnudsen droplet growth law as

$$
\frac{d r}{d t}=\lambda_{\mathrm{HK}} \Omega_{\mathrm{HK}}(p, T, y),
$$

where $\lambda_{\mathrm{HK}}$ will be referred to as the Hertz-Knudsen or free molecular growth parameter and $\Omega_{\mathrm{HK}}$, which depends on the local thermodynamic state, will be referred to as the free molecular or Hertz-Knudsen growth function.

Expressions for the growth parameters $\lambda_{C}$ and $\lambda_{\mathrm{H}-\mathrm{K}}$ and for the growth functions $\Omega_{C}$ and $\Omega_{\mathrm{HK}}$ are explicitly given in Appendix C. It should also be noted that the growth functions $\Omega_{C}$ and $\Omega_{\mathrm{HK}}$ are both of order of unity (indeed they are numerically close to each other). Rapid growth then suggests that both $\lambda_{C}$ and $\lambda_{\mathrm{HK}}$ are large compared to unity. Indeed we have

$$
\lambda_{C} \gg \lambda_{\mathrm{HK}} \gg 1 \text {. }
$$

From Eqs. (28) and (29) we obtain

$$
\frac{(d r / d t)_{C}}{(d r / d t)_{\mathrm{HK}}}=\frac{\mathrm{Nu}_{C}}{\mathrm{Nu}_{\mathrm{HK}}}=\frac{\lambda_{C} \Omega_{C}}{r \lambda_{\mathrm{HK}} \Omega_{\mathrm{HK}}} .
$$

Noting that $r$ is an increasing function of time (droplet growth), by virtue of Eq. (31), we can easily show that there exists an $\hat{r}$ given by the condition $\mathrm{Nu}_{C}=\mathrm{Nu}_{\mathrm{HK}}$, namely

$$
\hat{r} \equiv \frac{\lambda_{C} \Omega_{C}}{\lambda_{\mathrm{HK}} \Omega_{\mathrm{HK}}} .
$$

For droplets of size $r>\hat{r}$, the Hertz-Knudsen formula given by Eq. (28) yields higher growth rates whereas for droplets of size $r<\hat{r}$, the continuum growth formula given by Eq. (29) yields higher growth rates. In general, except for expansions under highly pressurized systems and aside from anomolies caused by nucleation theories near the coexistence line, initial growth of droplets takes place according to the Hertz-Knudsen formula $(\mathrm{Kn} \gg 1)$. As droplets grow deviations from the Hertz-Knudsen formula occur $[\mathrm{Kn}=O(1)]$. The continuum droplet growth regime can be reached asymptotically if relatively large droplets are formed at the local thermodynamic state, e.g., under high pressure conditions where $\mathrm{Kn} \ll 1$. It is then necessary to find expressions for droplet growth at arbitrary Knudsen number ranging from very small values to relatively large values compared to unity. Numerous expressions, called multirange expressions (e.g., see Gyarmathy ${ }^{25}$ ) and which interpolate between the Hertz-Knudsen and continuum formulas, have been suggested. One of the most widely used multirange expressions is given by Gyarmathy, ${ }^{25}$ which for the two-phase dispersed droplet regime considered herein takes the form

$$
\frac{\mathrm{Nu}_{G y}}{\mathrm{Nu}_{\mathrm{HK}}}=\frac{\sqrt{2 \pi} B_{M}^{\prime}\left(1-y_{v o}\right)}{4 \mathrm{Kn}}
$$

with

$$
B_{M}^{\prime}=\sqrt{\frac{2 R_{v}}{\pi R_{m}}} \alpha_{M}^{\prime} \mathrm{Sc}
$$


The Gyarmathy formula given by Eqs. (33)-(34) is just an interpolation formula between the Hertz-Knudsen and continuum regimes. A physical model based on the classical Langmuir model covering the intermediate regime at arbitrary Knudsen number has recently been proposed by Young $^{26,27}$ for both a pure vapor and a vapor in the presence of an inert gas. Young's results agree well especially with the numerical simulations of the Boltzmann equation ${ }^{28}$ and with the results of model kinetic equations. ${ }^{29,30}$ In addition the transition from the kinetic to diffusion controlled limit is satisfactorily recovered in Young's formula. ${ }^{27}$ However, Young's formula, as well as Gyarmathy's law given by Eqs. (33) - (34), are not suitable for analytical work which requires explicit determination of the droplet radius at arbitrary Knudsen number. In what follows we introduce an exactly solvable model for the droplet radius at arbitrary Knudsen number, which somewhat lies between the multirange growth rate expressions of Gyarmathy ${ }^{25}$ and Young. ${ }^{27}$

\section{An exactly solvable droplet growth model at arbitrary Knudsen number}

The initial growth of droplets usually takes place on clusters of critical size created in the vapor phase by homogeneous nucleation. For spherical droplets, depending on the critical radius $r_{\mathrm{cr}}^{\prime}$ and the mean free path $l^{\prime}$, the initial growth of droplets may obey different droplet growth formulas ranging from the free molecular to the continuum growth formulas. As mentioned before, if conditions are such that $\mathrm{Kn} \gg 1$ initially, droplet growth takes place according to the HertzKnudsen formula whereas if $\mathrm{Kn} \ll 1$ initially, the continuum regime growth formula holds. At intermediate Knudsen number, of the order of unity, a transitional growth formula is needed. Generally initial growth takes place according to the Hertz-Knudsen formula because of the small size of the critical radius; however, as droplets grow, a transitional character may emerge. In such a case, for a droplet created at a normalized time $\tau$, there exists a normalized transitional time $t_{1}$ (both normalized with respect to the expansion time $t_{r}^{\prime}$ ) where the Knudsen number reaches a universally assumed value $\mathrm{Kn}^{*}$ called the transition Knudsen number. For $t \leqslant t_{1}$ where $\mathrm{Kn} \geqslant \mathrm{Kn}^{*}$, the Hertz-Knudsen formula given by Eq. (29) is assumed to hold. Thus, for a droplet created at $\tau$, the normalized time $t_{1}$ can be evaluated from

$$
r^{*}\left(t_{1}\right) \equiv \frac{l\left(t_{1}\right)}{2 \mathrm{Kn}^{*}}=r_{\mathrm{cr}}(\tau)+\lambda_{\mathrm{HK}} \int_{\tau}^{t_{1}} \Omega_{\mathrm{HK}}(\eta) d \eta,
$$

where $r_{\mathrm{cr}}$ is the normalized critical radius of the droplet (with respect to $\left.r_{d}^{\prime}\right), r^{*}\left(t_{1}\right)$, and $l\left(t_{1}\right)$ are, respectively, the normalized radius and the mean free path (both normalized with respect to $r_{d}^{\prime}$ ) evaluated at $t=t_{1}$. In particular Eq. (35) relates $t_{1}$ to $\tau$ provided that a universal value for $\mathrm{Kn}^{*}$ is assumed. Despite the fact that one can satisfactorily use the multirange expressions of Gyarmathy ${ }^{25}$ and Young $^{27}$ with no reference whatsoever to $\mathrm{Kn}^{*}$, these formulas are not suitable for the analytical work to be pursued herein. We therefore introduce an exactly solvable droplet growth equation which apparently should satisfy the following requirements: (i) At $t \leqslant t_{1}$ or equivalently $\mathrm{Kn} \geqslant \mathrm{Kn}^{*}$, it should reduce to the Hertz-Knudsen formula.

(ii) The radius dependence of the growth rate, which is a consequence of the growth rate dependence on the Knudsen number, should be explicitly displayed and the resulting rate equation should be solvable to yield an explicit expression for the droplet growth radius for all times $t \geqslant t_{1}$.

(iii) For $\mathrm{Kn} \ll \mathrm{Kn}^{*}$, it should yield the continuum growth law of Eq. (28).

(iv) It should obey the general nondimensional growth expression (19), possibly in the quasisteady approximation, for the two-phase dispersed droplet model assumed.

(v) It should yield growth rates close to well-tested and generally accepted intermediate growth formulas such as those by Gyarmathy ${ }^{25}$ and Young. ${ }^{27}$ Fortunately the growth equation

$$
\frac{d r}{d t}=\frac{1}{\left[r-r^{*}\left(t_{1}\right)+\frac{\lambda_{C} \Omega_{C}^{*}}{\lambda_{\mathrm{HK}} \Omega_{\mathrm{HK}}^{*}}\right]} \lambda_{C} \Omega_{C}
$$

for $t \geqslant t_{1}$ with superscript $*$ denoting conditions evaluated at $t=t_{1}$, satisfies all the above requirements. Requirement (i) simply follows by evaluating Eq. (36) at $t=t_{1}$, where $\mathrm{Kn}=\mathrm{Kn}^{*}$ and $r=r^{*}\left(t_{1}\right)$ and by assuming that the HertzKnudsen formula of Eq. (29) holds for $t<t_{1}$. Requirement (ii) is satisfied by solving the differential equation (36) subject to the initial condition $r=r^{*}\left(t_{1}\right)$ at $t=t_{1}$. It then follows that

$$
r(\tau ; t)=r_{\mathrm{cr}}(\tau)+\lambda_{\mathrm{HK}} \int_{\tau}^{t} \Omega_{\mathrm{HK}}(\eta) d \eta
$$

for $t \leqslant t_{1}(\tau)$ (the free molecular regime), where $t_{1}(\tau)$ is given by Eq. (35) and

$$
\begin{aligned}
r(\tau ; t)= & r^{*}\left[t_{1}(\tau)\right]-\frac{\lambda_{C} \Omega_{C}^{*}}{\lambda_{\mathrm{HK}} \Omega_{\mathrm{HK}}^{*}} \\
& +\sqrt{\left(\frac{\lambda_{C} \Omega_{C}^{*}}{\lambda_{\mathrm{HK}} \Omega_{\mathrm{HK}}^{*}}\right)^{2}+2 \lambda_{C} \int_{t_{1}(\tau)}^{t} \Omega_{C}(\eta) d \eta}
\end{aligned}
$$

for $t \geqslant t_{1}(\tau)$ (the transition regime). In order to show that requirements (iii) $-(\mathrm{v})$ of Eq. (36) are satisfied, we cast Eq. (36) into the form

$$
\frac{\mathrm{Nu}}{\mathrm{Nu}_{C}}=\left[1+\left(\frac{\mathrm{Nu}_{C}^{*}}{\mathrm{Nu}_{\mathrm{HK}}^{*}}-1\right) \frac{\mathrm{Kn}}{\mathrm{Kn}^{*}} \frac{l^{*}}{l}\right]^{-1},
$$

where, as mentioned before, $\mathrm{Nu}_{C}^{*}=2, l \equiv l^{\prime} / r_{d}^{\prime}$ is the normalized mean free path of molecules in the gas mixture with $l^{\prime}$ given by the kinetic formula

$$
l^{\prime}=\frac{\eta^{\prime}}{2} \frac{\sqrt{2 \pi R_{m} T^{\prime}}}{p^{\prime}},
$$

and superscript $*$ refers to transition conditions at $t=t_{1}$. In Eq. (39) the ratios $l^{*} / l$ and $\mathrm{Nu}_{C}^{*} / \mathrm{Nu}_{\mathrm{HK}}^{*}$ are both of $O(1)$ in magnitude. It then follows that for $\mathrm{Kn} \ll \mathrm{Kn}^{*}$, Eq. (39) yields 


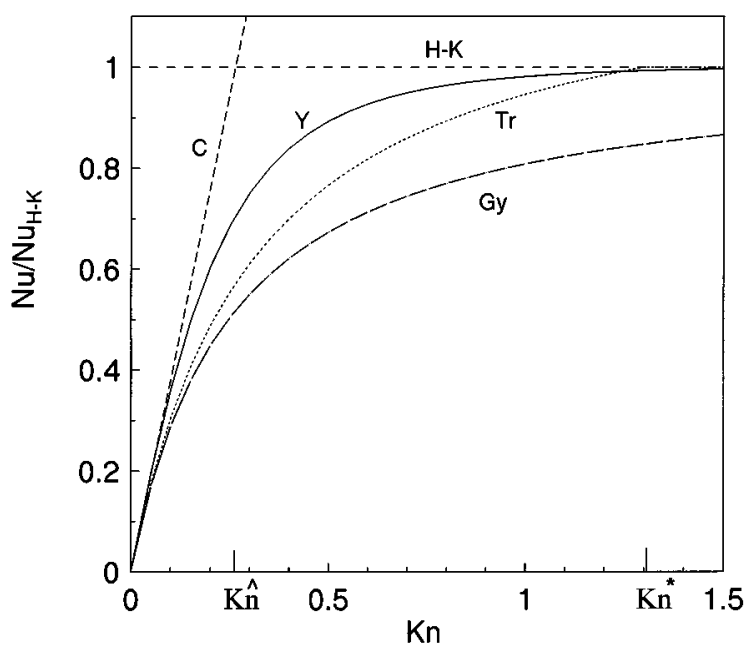

FIG. 1. Comparison of different droplet growth laws as a function of the Knudsen number. HK, Hertz-Knudsen growth law; $C$, continuum growth law; $Y$, Young's growth model; Gy, Gyarmathy's growth model; Tr, the proposed exactly solvable growth model $\left[\mathrm{Kn}^{*}\right.$ is the transition Knudsen number and $\hat{K} n$ is the Knudsen number of a droplet of normalized radius $\hat{r}$ given by Eq. (32)].

the continuum limit, i.e., $\mathrm{Nu} \approx \mathrm{Nu}_{c}=2$. Consequently Eq. (36) yields the continuum regime formula for $\mathrm{Kn} / \mathrm{Kn}^{*} \ll 1$; therefore, requirement (iii) is satisfied. Using the definition of the Schmidt number given by Eq. (22) and the definition of the mean free path given by Eq. (40) and taking into account the thermodynamic dependence of the diffusion coefficient $D^{\prime}$ (for most fluids it is approximately proportional to the square of the temperature and inversely proportional to the pressure), the transition growth Eq. (39) corresponding to the exactly solvable model of Eq. (36) can be written as

$$
\frac{\mathrm{Nu}}{\mathrm{Nu}_{C}}=\left[1+\left(\frac{\mathrm{Nu}_{C}^{*}}{\mathrm{Nu}_{\mathrm{HK}}^{*}}-1\right) \frac{\mathrm{Kn}^{*}}{\mathrm{Kn}^{*}} \frac{\mathrm{Sc}^{*}}{\mathrm{Sc}} \frac{1}{\xi}\right]^{-1},
$$

where $\xi$ is defined by

$$
\xi \equiv\left(\frac{T^{*}}{T}\right)^{\left(3-\gamma_{m}\right) / 2\left(\gamma_{m}-1\right)}
$$

with $\gamma_{m}$ denoting the initial adiabatic exponent of the carrier gas defined by $\gamma_{m} \equiv c_{p m} /\left(c_{p m}-1\right)$. In general $\xi$ is of order unity and varies numerically between 1 and 1.5 for most expansions where the Knudsen number Kn changes by orders of magnitude (the Schmidt number remains close to unity for most expansions). Thus the variation of $\xi$ can be neglected and $\xi$ itself may be treated as a constant, say with value unity during the expansion. With this in mind, Eq. (41) agrees with the nondimensional form (19) in the quasisteady approximation. This proves that Eq. (36) satisfies requirement (iv) to a good approximation.

Finally we compare the growth rates of the proposed transition model ( $\mathrm{Tr}$ ) with existing multirange expressions of Young and Gyarmathy. Figure 1 shows a comparison of the ratio $\mathrm{Nu} / \mathrm{Nu}_{\mathrm{HK}}$ for various models. All of the transition models (Gyarmathy, ${ }^{25}$ Young, ${ }^{27}$ and this model) approach the continuum model asymptotically for $\mathrm{Kn}<0.1$. For $\mathrm{Kn} \geqslant 1.31$

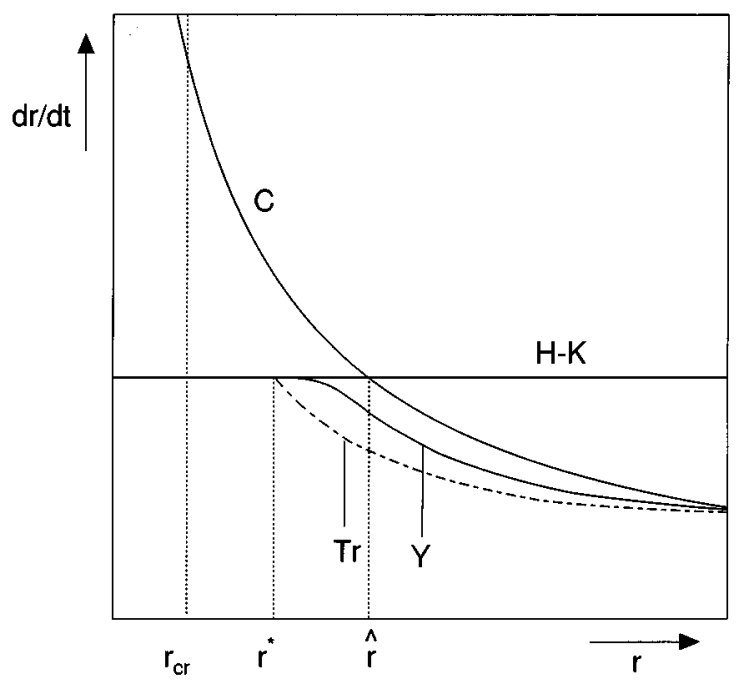

FIG. 2. Radius dependence of droplet growth rates in various models. HK, Hertz-Knudsen growth law; $C$, continuum growth law; $Y$, Young's growth model; $\mathrm{Tr}$, the proposed exactly solvable growth model $\left[r_{\mathrm{cr}}\right.$ is the normalized critical droplet radius, $r^{*}$ is the normalized droplet radius at transition, and $\hat{r}$ is the normalized droplet radius given by Eq. (32)].

Young's formula deviates from the Hertz-Knudsen formula negligibly (within 1\%). This inspires us to set the transition Knudsen number with value $\mathrm{Kn}^{*}=1.31$. Thus for $\mathrm{Kn} \geqslant \mathrm{Kn}^{*}=1.31$, the transition model is assumed to yield growth rates given precisely by the Hertz-Knudsen formula. The most commonly used Gyarmathy formula yields growth rates which are considerably smaller than those of Young's formula and the proposed model. The growth rates given by Eq. (36) of this model apparently lie between the growth rates given by Gyarmathy ${ }^{25}$ and Young ${ }^{27}$ for $\mathrm{Kn}<\mathrm{Kn}^{*}$, satisfying requirement (v). A comparison of the radius dependence of different droplet growth formulas is shown in Fig. 2. It can easily be seen that for droplets of normalized radius $r<\hat{r}$ with $\hat{r}$ given by Eq. (32), the continuum growth formula yields growth rates which are higher than those of the free molecular formula whereas for droplets of normalized radius $r>\hat{r}$, the Hertz-Knudsen formula yields the highest rates. Young's formula yields rates which are lower than both of the free molecular and continuum growth rates. The proposed transition model, for intermediate Knudsen numbers, yields even lower growth rates (the rates given by Gyarmathy's formula, although not shown, would yield the lowest rates for fixed size of all the growth rates discussed). For sufficiently large droplets all of these transition models seem to approach the continuum growth law.

\section{The condensation rate equation}

The condensation rate equation is constructed from a nucleation rate equation and a droplet growth law. Denoting the radius of a spherical droplet at $t^{\prime}$ created at $\tau^{\prime}$ by $r^{\prime}\left(\tau^{\prime} ; t^{\prime}\right)$, the condensate molar fraction $y$ at $t^{\prime}$ can be written as 


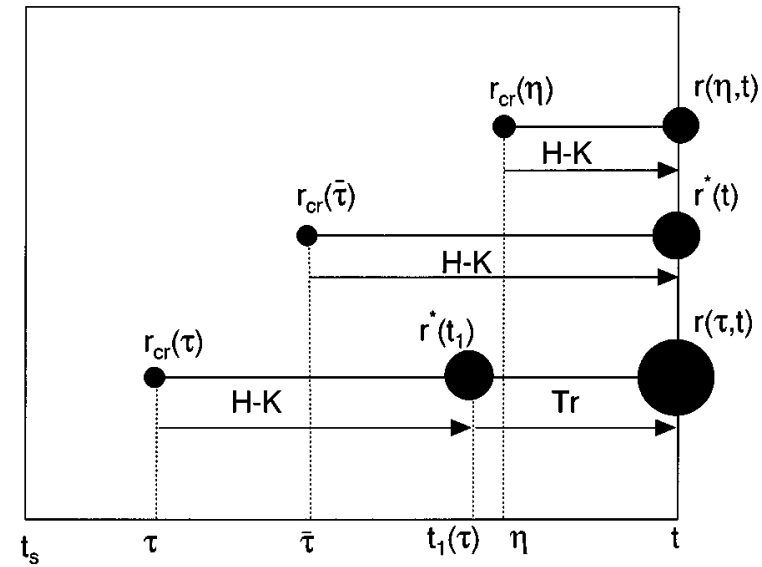

FIG. 3. Droplet growth history of groups of droplets of various sizes at any given instant $t$ ( $\bar{\tau}$ is the normalized time at which the groups of droplets in transition at $t$ are created and $r^{*}$ is the normalized droplet radius at transition).

$$
y\left(t^{\prime}\right)=\frac{4}{3} \pi \overrightarrow{n_{l}} \int_{t_{s}^{\prime}}^{t^{\prime}}\left[r^{\prime}\left(\tau^{\prime} ; t^{\prime}\right)\right]^{3} \frac{J^{\prime}\left(\tau^{\prime}\right)}{n^{\prime}\left(\tau^{\prime}\right)} d \tau^{\prime} .
$$

By substituting from Eq. (14) for $J^{\prime}$ and using normalized variables together with the definition of radius normalization constant $r_{d}^{\prime}$ given by Eq. (27), the condensation rate Eq. (43) takes the form

$$
y(t)=\int_{t_{s}}^{t}[r(\tau ; t)]^{3} \frac{\Sigma(\tau)}{n(\tau)} \exp \left[-K^{-1} B(\tau)\right] d \tau .
$$

The functions $\Sigma$ and $B$ and the nucleation parameter $K$ are presumably to be obtained from the particular theory of homogeneous nucleation to be employed. The normalized radius $r(\tau ; t)$, on the other hand, depends on the particular regime where the droplet grows. As mentioned earlier, in general droplets initially grow in the Hertz-Knudsen regime. If the duration of the expansion is sufficiently large so that at least a group of droplets reach Knudsen numbers lower than the transition Knudsen number $\mathrm{Kn}^{*}$, then the normalized radius $r(\tau ; t)$ of this group of droplets cannot be solely determined by the Hertz-Knudsen formula given by Eq. (29). Usually, for sufficiently long duration of the expansion, there will be groups of droplets that grow according to different growth laws. In particular there will be a group of droplets at normalized time $t$ which is just entering the transition regime with Knudsen number $\mathrm{Kn}^{*}$ and created at $\bar{\tau}$ defined by

$$
t=t_{1}(\vec{\tau}) .
$$

For any sufficiently large $t, \bar{\tau}$ can be obtained by solving the functional Eq. (35) with $\tau$ replaced by $\bar{\tau}$. It can easily be shown that groups of droplets created at $\tau<\bar{\tau}$ will grow according to the transition regime multirange expressions whereas groups of droplets created at $\tau>\bar{\tau}$ will grow according to the Hertz-Knudsen formula of Eq. (29). Figure 3 shows three different groups of droplets with different growth rates at $t$. The group of droplets created at $\tau<\bar{\tau}$ with normalized critical radius $r_{\mathrm{cr}}(\tau)$ enter the transition regime at $t_{1}(\tau)<t$. For times greater than $t_{1}(\tau)$ the growth of these droplets proceed in the transition regime. On the other hand the group of droplets created at $\eta>\bar{\tau}$ still grow according to the Hertz-Knudsen formula. The limiting group of droplets created at $\bar{\tau}$ enter the transition regime at just the instant $t$ considered. Thus the expression for $r(\tau ; t)$ cannot in general be given by a single formula over the whole range of Knudsen numbers. Let $\bar{\tau}=\tau^{*}(t)$ be a function defined by the inverse of relation (45). Utilizing Eqs. (37) and (38) for the normalized radius $r(\tau ; t)$ in the exactly solvable model proposed, the condensation rate equation (44) for the molar fraction $y(t)$ then assumes the general form

$$
\begin{aligned}
y(t)= & \int_{t_{s}}^{t}\left[r_{\mathrm{cr}}(\tau)+\lambda_{\mathrm{HK}} \int_{\tau}^{t} \Omega_{\mathrm{HK}}(\eta) d \eta\right]^{3} \frac{\Sigma(\tau)}{n(\tau)} \\
& \times \exp \left[-K^{-1} B(\tau)\right] d \tau
\end{aligned}
$$

for all $t<\tau^{*}(t)$ and

$$
\begin{aligned}
y(t)= & \int_{t_{s}}^{\tau^{*}(t)}\left[r_{\mathrm{cr}}(\tau)+\lambda_{\mathrm{HK}} \int_{\tau}^{t_{1}(\tau)} \Omega_{\mathrm{HK}}(\eta) d \eta-\frac{\lambda_{C} \Omega_{C}^{*}}{\lambda_{\mathrm{HK}} \Omega_{\mathrm{HK}}^{*}}\right. \\
& +\sqrt{\left.\left(\frac{\lambda_{C} \Omega_{C}^{*}}{\lambda_{\mathrm{HK}} \Omega_{\mathrm{HK}}^{*}}\right)^{2}+2 \lambda_{C} \int_{t_{1}(\tau)}^{t} \Omega_{C}(\eta) d \eta\right]^{3} \frac{\Sigma(\tau)}{n(\tau)}} \\
& \times \exp \left[-K^{-1} B(\tau)\right] d \tau+\int_{\tau^{*}(t)}^{t}\left[r_{\mathrm{cr}}(\tau)+\lambda_{\mathrm{HK}}\right. \\
& \left.\times \int_{\tau}^{t} \Omega_{\mathrm{HK}}(\eta) d \eta\right]^{3} \frac{\Sigma(\tau)}{n(\tau)} \exp \left[-K^{-1} B(\tau)\right] d \tau
\end{aligned}
$$

for all $t>\tau^{*}(t)$.

In cases when at no instant the condition $t>\tau^{*}(t)$ is satisfied, we have the strictly Hertz-Knudsen regime. The condensation rate equation is then given by Eq. (46) for all $t$. It may also happen, e.g., during expansions with very high initial pressures, that all droplets are created in a continuum environment for which the continuum growth formula of Eq. (28) holds (strictly continuum regime). In this case the normalized radius $r(\tau ; t)$ takes the form

$$
r(\tau ; t)=\sqrt{r_{\mathrm{cr}}^{2}(\tau)+2 \lambda_{C} \int_{\tau}^{t} \Omega_{C}(\eta) d \eta}
$$

for all $t$ during the expansion. The condensation rate equation for the molar fraction $y$ in this strictly continuum regime then reduces to the equation

$$
\begin{aligned}
y(t)= & \int_{t_{s}}^{t}\left[r_{\mathrm{cr}}^{2}(\tau)+2 \lambda_{C} \int_{\tau}^{t} \Omega_{C}(\eta) d \eta\right]^{3 / 2} \frac{\Sigma(\tau)}{n(\tau)} \\
& \times \exp \left[-K^{-1} B(\tau)\right] d \tau
\end{aligned}
$$

for all $t$

The general condensation rate equation given by Eqs. (46) and (47) is a nonlinear Volterra integral equation coupled to the governing equations discussed in Sec. II A. Its analytical solution, though possible in principle, is difficult to obtain. Despite the fact that the unknown thermodynamic functions $r_{\mathrm{cr}}, \Omega_{C}, \Omega_{\mathrm{HK}}, \Sigma, B$ and the unknown parameters 


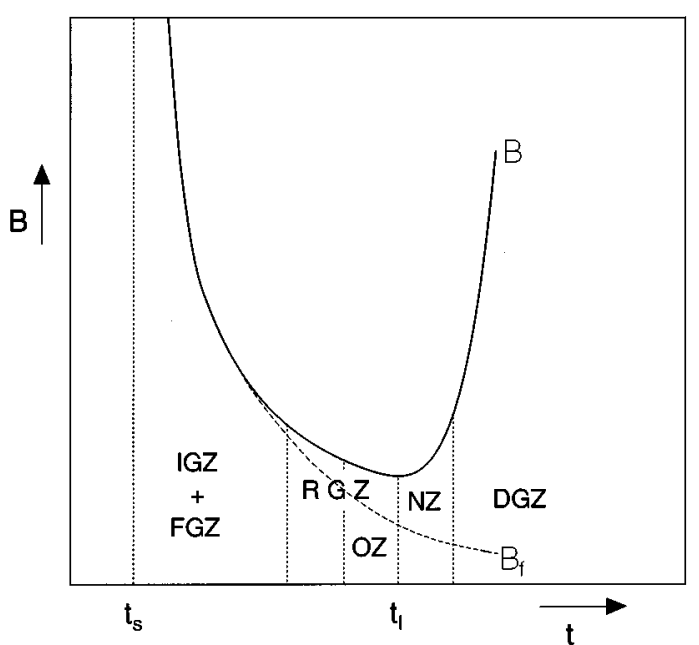

FIG. 4. Variation of the normalized activation function $B$ with normalized time and the distinct condensation zones $\left(B_{f}\right.$ represents the normalized frozen activation function, $t_{s}$ is the normalized time at which saturation is reached, and $t_{l}$ is the turning point of the activation function $B$ ).

$K, \zeta^{\prime}, \lambda_{C}, \lambda_{\mathrm{HK}}$ need to be identified by the appropriate nucleation and growth equations to be employed, one in addition has to solve Eq. (35) with $\tau$ replaced by $\bar{\tau}$ to obtain Eq. (45) and then invert it to get the function $\tau^{*}(t)$. Granted this first step is taken, we can exploit the fact that the nucleation parameter $K$ is small as compared to unity (corresponding to relatively large nucleation period during expansion) and that the growth parameters $\lambda_{C}$ and $\lambda_{\mathrm{HK}}$ are large as compared to unity (corresponding to rapid growth of droplets) in order to obtain an analytical solution of Eqs. (46) and (47). In this way we solve Eqs. (46) and (47) [and also the limiting Eq. (49)] by Laplace's method ${ }^{31,32}$ in the limit as

$$
K \rightarrow 0 \text { and } \lambda_{C}, \lambda_{\mathrm{HK}} \rightarrow \infty \text { with } \frac{\lambda_{C}}{\lambda_{\mathrm{HK}}}=\text { finite. }
$$

We discuss these solutions below for different droplet growth regimes.

\section{ASYMPTOTIC SOLUTION OF THE CONDENSATION RATE EQUATION IN DIFFERENT DROPLET GROWTH REGIMES}

The asymptotic solution of the condensation rate equation in different droplet growth regimes is constructed in the limit as $K \rightarrow 0$ and $\lambda_{C}, \lambda_{\mathrm{HK}} \rightarrow \infty$. In all of the different growth regimes the behavior of the activation function $B=B(t)$ distinguishes the distinct condensation zones during the expansion. A typical variation of the activation function $B$ during a continuous expansion is shown in Fig. 4. For $t \leqslant t_{s}$, with $t_{s}$ denoting the time when the condensable vapor becomes saturated, $B$ is infinite (vanishing nucleation rates). For $t>t_{s}$, the vapor expands in metastable state resulting in a decreasing behavior of the activation function $B$. At some instant $t_{l}>t_{s}$ during the expansion, $B$ exhibits an absolute minimum given by

$$
\left(\frac{d B}{d t}\right)_{t=t_{l}}=0
$$

corresponding to a maximum nucleation rate. The instant this occurs, i.e., the point where $t=t_{l}$ is called the relative onset point.

In the interval $t_{s} \leqslant t \leqslant t_{l}$ four physically distinct condensation zones (only two are asymptotically distinct) can be identified. These are the initial growth zone (IGZ), further growth zone (FGZ), rapid growth zone (RGZ) and onset zone $(\mathrm{OZ})$. The initial and further growth zones are defined as the zones where $d B / d t=O(1)$. They are distinguished physically in such a way that in the initial growth zone (IGZ), $B=B_{f}$ where $B_{f}$ denotes the frozen activation function with $y \equiv 0$. In this zone all of the flow variables plus their derivatives are given by their frozen values. In the further growth zone (FGZ), where $d B / d t$ is still of $O(1)$ in magnitude, considerable deviations in the time derivatives of the flow variables from their isentropic values may occur despite the fact that deviations of the variables themselves from their isentropic values are negligibly small. The rapid growth (RGZ) and onset (OZ) zones are defined as the zones where $d B / d t$ diminishes to $O\left(K^{1 / 2}\right)$ as $K \rightarrow 0$. The onset zone (OZ), which contains the empirical onset of condensation, is in fact embedded in the rapid growth zone (RGZ). In particular the relative onset point $t_{l}$, where maximum nucleation is practically reached, marks the end of the onset zone.

For $t>t_{l}$, vapor depletion can no longer be neglected and two more physically and asymptotically distinct zones are identified from the asymptotic solution of the rate equation. These are the nucleation zone with growth (NZ) where both nucleation and droplet growth are important, and the droplet growth zone (DGZ) which is dominated by droplet growth. The nucleation zone with growth starts at $t=t_{l}$ and extends until nucleation rates are practically negligible. Relaxation of the nonequilibrium two-phase mixture to saturated equilibrium states takes place in the droplet growth zone (DGZ). These characteristic condensation zones are unique in all of the different droplet growth regimes; however, different droplet growth rates lead to different asymptotic expressions in each zone. It is important to note that the asymptotic expressions for the condensate molar fraction hold independent of any particular homogeneous nucleation theory to be employed. Except for IGZ, where the normalized temperature $T$ and the normalized molar density $n$ of the mixture are given by their isentropic values and the condensation rate equation for the molar fraction is decoupled from the rest of the governing equations, the asymptotic expressions for the condensate molar fraction $y(t)$ in distinct condensation zones for each droplet growth regime are combined with the solution of the governing Eqs. (11)-(13) to yield the temperature $T(t)$ and the molar density $n(t)$ by iteration [Eq. (13) for $\phi(t)$ is solved by quadrature utilizing the asymptotic expressions for $y(t)$ in distinct condensation zones in each droplet growth regime]. Thus the complete solution is obtained provided that we solve the condensation rate equation for the molar fraction $y$ in distinct condensation 
zones in each growth regime to obtain the corresponding asymptotic expressions.

\section{A. Free molecular (Hertz-Knudsen) regime}

In the free molecular regime where $\mathrm{Kn} \gg 1$, the condensation rate equation for the molar fraction $y$ assumes the form of Eq. (46) for all times during the expansion. The asymptotic solution of the rate Eq. (46) in the double limit as $K \rightarrow 0$ and $\lambda_{\mathrm{HK}} \rightarrow \infty$ has already been carried out in a different investigation ${ }^{16}$ using Laplace's method. ${ }^{31,32}$ The free molecular asymptotic expressions in distinct condensation zones can be found in Ref. 16 and need not be reproduced herein. (In the asymptotic expressions of Ref. 16 the condensate mass fraction $g$ and the normalized mass density $\rho$ should, respectively, be replaced by the molar fraction $y$ and the normalized molar density $n$. In addition $\lambda=\lambda_{\mathrm{HK}}$ and $\Omega=\Omega_{\mathrm{HK}}$.)

\section{B. Transition regime}

The condensation rate equation in the transition regime based on the exactly solvable model of Sec. II C and given by Eqs. (46) and (47) is in general difficult to solve asymptotically in the limit as $K \rightarrow 0$ and $\lambda_{C}, \lambda_{\mathrm{HK}} \rightarrow \infty$ with $\lambda_{C} / \lambda_{\mathrm{HK}}=$ finite. A serious difficulty arises in determining $\tau^{*}(t)$ by inverting Eq. (45). This problem can be easily bypassed (although solvable in principle) if we assume that the duration for which droplets grow in the initial HertzKnudsen regime is small compared to the remaining period. In other words we assume that droplets of critical size enter the transition regime as soon as they are created. With this assumption, which is reasonable for many practical applications, we obtain

$$
\tau^{*}(t)=t,
$$

i.e., the function $\tau^{*}$ reduces to the identity function. The condensation rate Eq. (47) for the molar fraction $y$ in this regime then takes the simplified form

$$
\begin{aligned}
y(t)= & \int_{t_{s}}^{t}\left\{r_{\mathrm{cr}}(\tau)-\frac{\lambda_{C} \Omega_{C}(\tau)}{\lambda_{\mathrm{HK}} \Omega_{\mathrm{HK}}(\tau)}\right. \\
& \left.+\sqrt{\left[\frac{\lambda_{C} \Omega_{C}(\tau)}{\lambda_{\mathrm{HK}} \Omega_{\mathrm{HK}}(\tau)}\right]^{2}+2 \lambda_{C} \int_{\tau}^{t} \Omega_{C}(\eta) d \eta}\right\}^{3} \frac{\Sigma(\tau)}{n(\tau)} \\
& \times \exp \left[-K^{-1} B(\tau)\right] d \tau
\end{aligned}
$$

for all $t \geqslant t_{s}$. Using Laplace's method ${ }^{31,32}$ the asymptotic solution of Eq. (53) in the limit as $K \rightarrow 0$ and $\lambda_{C}, \lambda_{\mathrm{HK}} \rightarrow \infty$ with $\lambda_{C} / \lambda_{\mathrm{HK}}=$ finite, yields the asymptotic expressions in distinct condensation zones of the transition regime, as exhibited in Appendix A.

\section{Continuum regime}

In some extreme situations such as those encountered under highly pressurized conditions, droplets of critical size may be created in a continuum environment $(\mathrm{Kn} \ll 1)$. In such a case droplet growth can be evaluated solely by the con- tinuum growth formula of Eq. (28). The condensate rate equation for the molar fraction $y$ is then given by Eq. (49). The asymptotic expressions in distinct condensation zones of this regime then follow by Laplace's method ${ }^{31,32}$ in the double limit as $K \rightarrow 0$ and $\lambda_{C} \rightarrow \infty$, as discussed in detail in Appendix B.

\section{APPLICATIONS OF THE ASYMPTOTIC THEORY AND COMPARISON WITH NUMERICAL SIMULATIONS}

The asymptotic solution presented in the preceding section can be directly applied to predict the nucleation rate $J^{\prime}$, the condensate molar fraction $y$, and the flow field (the molar density $n^{\prime}$ and the temperature $T^{\prime}$ ) during a continuous expansion provided that the pressure variation is assumed (or measured). The thermodynamic properties such as surface tension, diffusion coefficient, mass accommodation coefficient, latent heat, etc. are given together with the identification of the thermodynamic functions and parameters that enter the normalized nucleation and droplet growth equations. The pressure signal will be assumed to be of the form

$$
p^{\prime}\left(t^{\prime}\right)=p_{0}^{\prime}\left[0.05+0.95 \exp \left(-\frac{t^{\prime}}{t_{2}^{\prime}}\right)\right]
$$

where $p_{0}^{\prime}$ denotes the total initial pressure of the mixture and $t_{2}^{\prime}$ is a measure of the relaxation time for the total pressure $p^{\prime}$ to drop to $5 \%$ of its initial value $p_{0}^{\prime}$. In particular the expansion time $t_{r}^{\prime}$ (normalization constant for the time coordinate) of Eq. (9) becomes

$$
t_{r}^{\prime}=\frac{20}{19} t_{2}^{\prime}
$$

For practical applications of the theory we consider the homogeneous condensation of water vapor in nitrogen during continuous expansions in cloud chambers. For the homogeneous nucleation of water vapor in the range of temperatures considered, we choose the classical nucleation theory ${ }^{17,18}$ which yields nucleation rates within one or two orders of magnitude in comparison with experiments (we herein prefer the classical nucleation equation due to its simplicity in spite of the fact that the recent semiphenomenological theories ${ }^{22-24}$ can improve the classical rates by an order of magnitude in the range of temperatures considered). The normalizations of the classical nucleation theory and of the continuum and free molecular growth laws, from which the normalized thermodynamic functions $\Sigma, B, \Omega_{\mathrm{HK}}, \Omega_{C}$, $r_{\text {cr }}$, the nucleation parameter $K$ and the growth parameters $\lambda_{\mathrm{HK}}$ and $\lambda_{C}$ can be identified, are given in Appendix C together with certain thermodynamic properties of water vapor and nitrogen which enter the asymptotic theory. With the results of Appendix C, we can calculate certain characteristics of homogeneous condensation, such as the shape of the nucleation curve and the condensate mass fraction during 


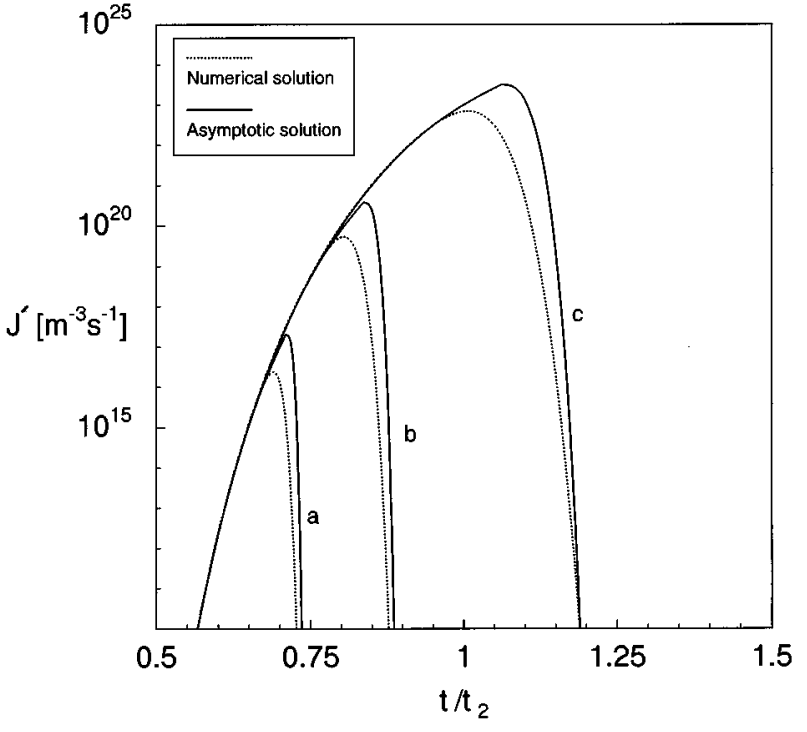

FIG. 5. Comparison of nucleation rates obtained by numerical simulations and asymptotic solution for the expansion of water vapor in nitrogen with initial conditions $p_{0}^{\prime}=1 \mathrm{bar},\left(p_{v}^{\prime}\right)_{0}=1000 \mathrm{~Pa}$, and $T_{0}^{\prime}=295 \mathrm{~K}$ in the free molecular (Hertz-Knudsen) regime at different expansion times. (a) $t_{2}^{\prime}=10^{-2} \mathrm{~s}$. (b) $t_{2}^{\prime}=10^{-3} \mathrm{~s}$. (c) $t_{2}^{\prime}=10^{-4} \mathrm{~s}$.

expansions, by the asymptotic theory for different initial conditions [for the results presented we fix the initial temperature by $T_{0}^{\prime}=295 \mathrm{~K}$ and the initial partial water vapor pressure by $\left(p_{v}^{\prime}\right)_{0}=1000 \mathrm{~Pa}$ and vary the initial total mixture pressure between 1 and 50 bars]. In addition we simulate the results for the same conditions numerically for comparison with the results of the asymptotic solution (details of the method used for numerical simulations can be found in the dissertation of Muitjens ${ }^{33}$ ).

Figure 5 shows a comparison of numerically and asymptotically achieved nucleation rates in the Hertz-Knudsen regime for different expansion times with an initial pressure $p_{0}^{\prime}=1$ bar. There is an excellent agreement between numerical and asymptotic nucleation rates in the initial (IGZ) and further growth (FGZ) zones. Deviations begin to occur somewhere in the rapid growth zone (RGZ) due to the second order asymptotic solution (for the expansions considered third order derivatives of the activation function $B$ seem to be important in this zone, especially near the turning point $t_{l}$ due to the steep change in $B$ ). The maximum nucleation rates are somewhat an order of magnitude higher in the asymptotic solution than in the numerical solution. Consequently the nucleation rates drop in the nucleation zone with growth (NZ) faster in the asymptotic solution than the numerical one with an eventual approach toward each other. The differences decrease as the expansion time $t_{2}^{\prime}$ is increased. Figure 6 shows a comparison of the asymptotic and numerical molar fractions in the Hertz-Knudsen regime for the same expansion. Deviations in $y$ also occur in the rapid growth zone (RGZ) where the asymptotic predictions fall below the numerical ones due to the reasons mentioned above, i.e., the second order approximation to the activation function $B$ in the asymptotic theory. Consequently a steeper variation of $y$

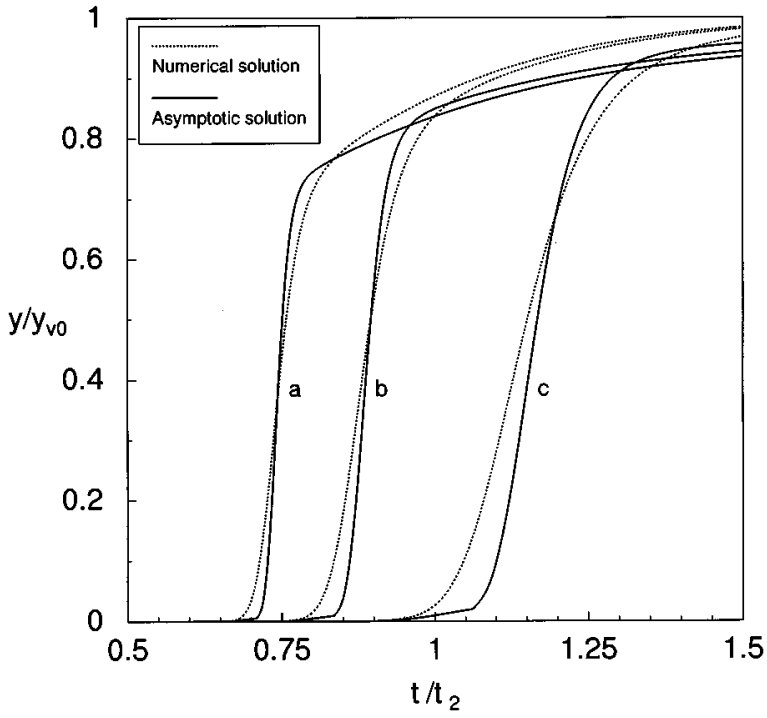

FIG. 6. Comparison of molar fractions obtained by numerical simulations and asymptotic solution for the expansion of water vapor in nitrogen with initial conditions $p_{0}^{\prime}=1 \mathrm{bar},\left(p_{v}^{\prime}\right)_{0}=1000 \mathrm{~Pa}$ and $T_{0}^{\prime}=295 \mathrm{~K}$ in the free molecular (Hertz-Knudsen) regime at different expansion times. (a) $t_{2}^{\prime}=10^{-2} \mathrm{~s}$. (b) $t_{2}^{\prime}=10^{-3} \mathrm{~s}$. (c) $t_{2}^{\prime}=10^{-4} \mathrm{~s}$.

in the nucleation zone with growth (NZ) is observed in the asymptotic theory. It is important to mention that there is no discontinuity in the derivative of $y$ at $t=t_{l}$, where RGZ and $\mathrm{NZ}$ overlap. (The very sharp change in $d y / d t$ at $t=t_{l}$ in the asymptotic solution of Fig. 6 should thereby not be interpreted as a discontinuous change. The almost discontinuous behavior of $d y / d t$ at $t=t_{l}$ that appears to the eye is caused by the plotter's linear approximation between neighboring points of the turning point $t_{l}$ where a very steep change in $y$ occurs.) Once again the differences somewhat seem to diminish in DGZ downstream of NZ for smaller expansion times $t_{2}^{\prime}$.

For intermediate initial pressures $p_{0}^{\prime}$ (e.g., $p_{0}^{\prime}=10$ bar) droplet growth takes place in the transition regime. This case represents an opportunity to compare predictions of different droplet growth regimes, namely the Hertz-Knudsen, continuum and transition regimes. In the transition regime we use the exactly solvable transition model $(\mathrm{Tr})$ introduced in Sec. II C in the asymptotic theory whereas the widely used interpolation formula of Gyarmathy (Gy) in numerical simulations. A comparison of the asymptotic and numerical nucleation rates obtained by the Hertz-Knudsen and transition ( $\mathrm{Tr}$ in the asymptotic solution, Gy in the numerical solution) is shown in Fig. 7. Despite excellent qualitative agreement, quantitative differences between numerical and asymptotic solutions occur (especially in RGZ and NZ) due to similar reasons discussed above. The maximum nucleation rates of numerical and asymptotic solutions seem to be within an order of magnitude [a better agreement is achieved between the transition model ( $\mathrm{Tr}$ ) and Gyarmathy's formula $(G y)]$. The comparison of the nucleation rates in this intermediate regime for the Hertz-Knudsen (HK), continuum (C) and transition (Tr) models by the asymptotic solution is 


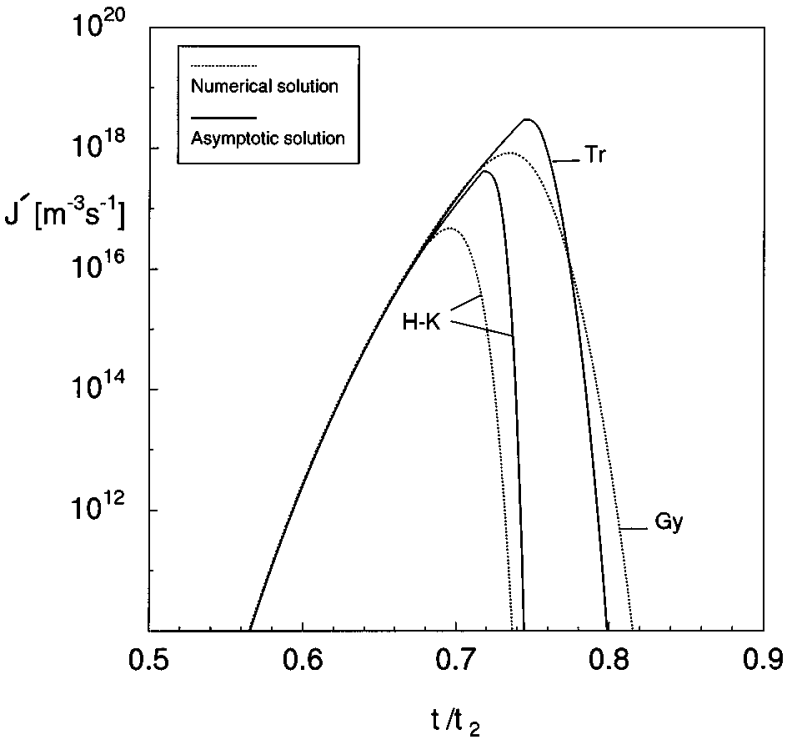

FIG. 7. Comparison of nucleation rates obtained by numerical simulations and asymptotic solution for the expansion of water vapor in nitrogen with expansion time $t_{2}^{\prime}=10^{-2} \mathrm{~s}$ and initial conditions $p_{0}^{\prime}=10 \mathrm{bar},\left(p_{v}^{\prime}\right)_{0}=1000$ $\mathrm{Pa}$ and $T_{0}^{\prime}=295 \mathrm{~K}$ in the free molecular (HK), Gyarmathy (Gy), and proposed exactly solvable ( $\mathrm{Tr}$ ) models (in the transition regime the Gyarmathy model is used for numerical simulations whereas the proposed exactly solvable model is used for asymptotic predictions).

shown in Fig. 8. The Tr model yields the highest and the HK model the lowest maximum nucleation rates for this case. A similar comparison by the numerical solution (where the $\mathrm{Tr}$ model is replaced by the Gy formula) yields similar qualitative and quantitative behavior as shown in Fig. 9. Finally a comparison of the results for the molar fraction obtained by

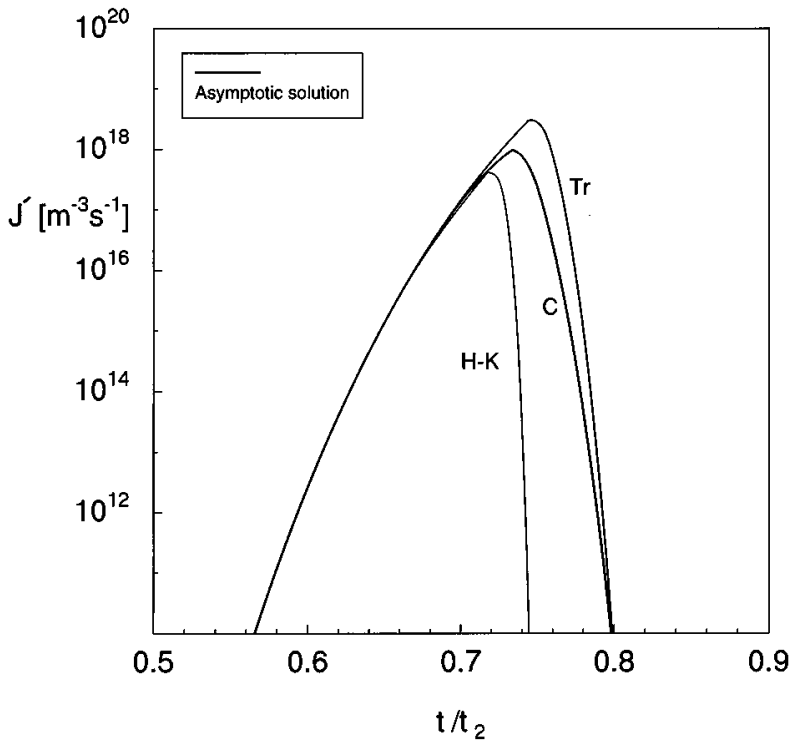

FIG. 8. Comparison of nucleation rates obtained by the asymptotic solution for the expansion of water vapor in nitrogen with expansion time $t_{2}^{\prime}=10^{-2} \mathrm{~s}$ and initial conditions $p_{0}^{\prime}=10 \mathrm{bar},\left(p_{v}^{\prime}\right)_{0}=1000 \mathrm{~Pa}$ and $T_{0}^{\prime}=295 \mathrm{~K}$ in different growth regimes (HK, Hertz-Knudsen growth law; $C$, continuum growth law; $\mathrm{Tr}$, the proposed exactly solvable growth model).

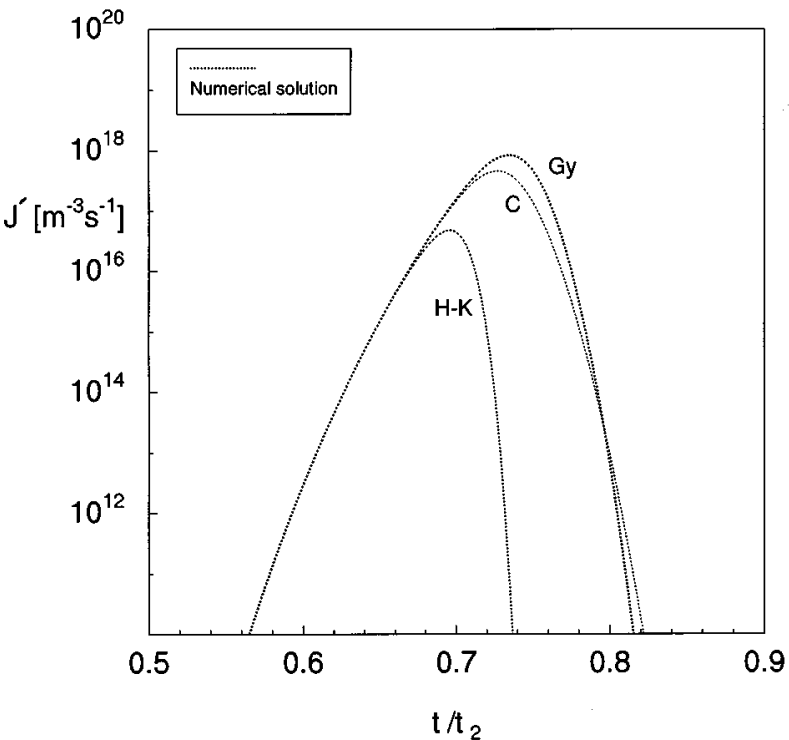

FIG. 9. Comparison of nucleation rates obtained by numerical simulations for the expansion of water vapor in nitrogen with expansion time $t_{2}^{\prime}=10^{-2} \mathrm{~s}$ and initial conditions $p_{0}^{\prime}=10 \mathrm{bar},\left(p_{v}^{\prime}\right)_{0}=1000 \mathrm{~Pa}$, and $T_{0}^{\prime}=295 \mathrm{~K}$ in different growth regimes (HK, Hertz-Knudsen growth law; $C$, continuum growth law; Gy, Gyarmathy's growth model).

the asymptotic and numerical solutions for different growth equations (HK, $C$, Tr for the asymptotic solution and Gy for the numerical solution) is given in Fig. 10. Differences between asymptotic and numerical solutions occur due to similar reasons discussed above. A better agreement between the asymptotic and numerical solutions is reached in this case for

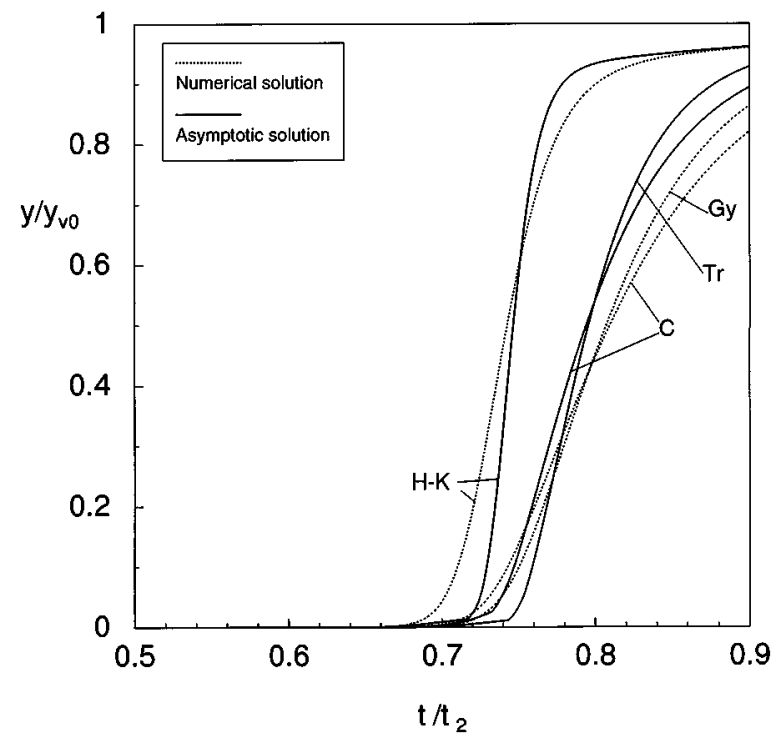

FIG. 10. Comparison of molar fractions obtained by the asymptotic solution and numerical simulations for the expansion of water vapor in nitrogen with expansion time $t_{2}^{\prime}=10^{-2} \mathrm{~s}$ and initial conditions $p_{0}^{\prime}=10 \mathrm{bar},\left(p_{v}^{\prime}\right)_{0}=1000$ $\mathrm{Pa}$ and $T_{0}^{\prime}=295 \mathrm{~K}$ in different growth regimes (HK, Hertz-Knudsen growth law; $C$, continuum growth law; Gy, Gyarmathy's growth model; Tr, the proposed exactly solvable growth model). 


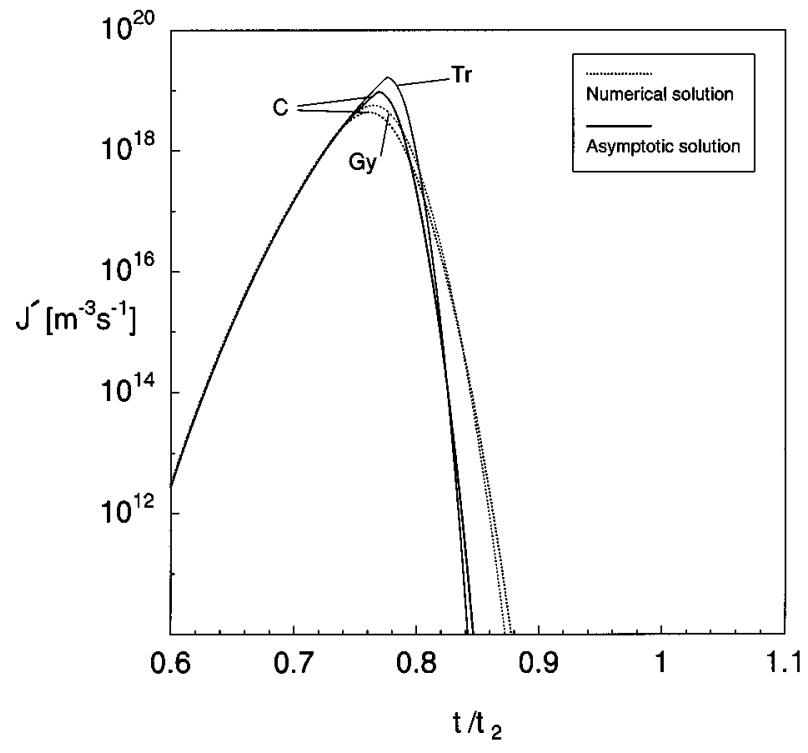

FIG. 11. Comparison of nucleation rates obtained by the asymptotic solution and numerical simulations for the expansion of water vapor in nitrogen with expansion time $t_{2}^{\prime}=10^{-2} \mathrm{~s}$ and initial conditions $p_{0}^{\prime}=50$ bar, $\left(p_{v}^{\prime}\right)_{0}=1000 \mathrm{~Pa}$, and $T_{0}^{\prime}=295 \mathrm{~K}$ in different growth regimes $(C$, continuum growth law; Gy, Gyarmathy's growth model; Tr, the proposed exactly solvable growth model).

the continuum $(C)$ and Gyarmathy $(\mathrm{Gy})$ and proposed transition $(\mathrm{Tr})$ formulas.

Finally for high initial pressures $\left(p_{0}^{\prime}=50 \mathrm{bar}\right)$ the transition (Tr), Gyarmathy (Gy), and continuum $(C)$ growth laws are compared by the numerical and asymptotic solutions. A comparison of nucleation rates is plotted in Fig. 11, whereas a comparison of the molar fractions can be found in Fig. 12. Despite the differences previously discussed between asymptotic and numerical solutions, a better quantitative agreement is reached for this case. This demonstrates that, for higher pressures, the differences between the results of the second order asymptotic solution and of the numerical simulations seem to decrease.

\section{CONCLUSIONS}

Homogeneous condensation of a condensable vapor in a carrier gas in expansion cloud chambers is investigated in detail for different droplet growth regimes (free molecular, transition, and continuum) depending on the initial vapor pressure with fixed initial temperature and initial partial vapor pressure. In particular an exactly solvable droplet growth model in the transition regime at arbitrary Knudsen number is presented. This model yields growth rates that lie in between those given by Gyarmathy's ${ }^{25}$ and Young's ${ }^{27}$ droplet growth formulas, the latter ones being the most widely used ones in the literature. The condensation rate equation for the molar fraction constructed from an arbitrary steady-state nucleation rate equation together with different droplet growth laws (free molecular, continuum, transition) is then solved asymptotically in the limit of relatively large nucleation time $(K \rightarrow 0)$ and small droplet growth time $\left(\lambda_{\mathrm{HK}}\right.$, $\left.\lambda_{C} \rightarrow \infty\right)$ and the distinct condensation zones together with the

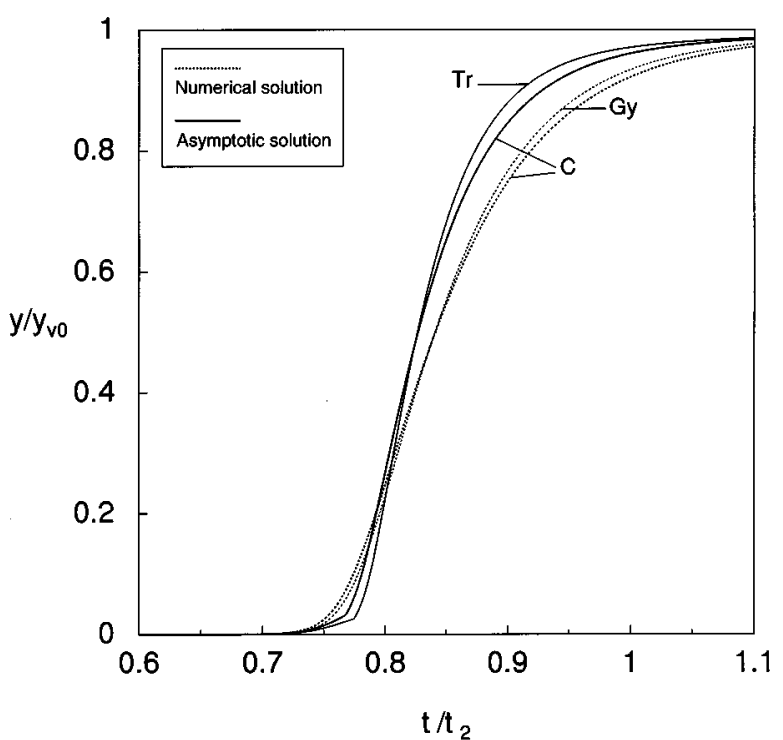

FIG. 12. Comparison of molar fractions obtained by the asymptotic solution and numerical simulations for the expansion of water vapor in nitrogen with expansion time $t_{2}^{\prime}=10^{-2} \mathrm{~s}$ and initial conditions $p_{0}^{\prime}=50 \mathrm{bar},\left(p_{v}^{\prime}\right)_{0}=1000$ $\mathrm{Pa}$, and $T_{0}^{\prime}=295 \mathrm{~K}$ in different growth regimes $(C$, continuum growth law; Gy, Gyarmathy's growth model; Tr, the proposed exactly solvable growth model).

corresponding asymptotic expressions of the condensate molar fraction are obtained in each growth regime. Nucleation rates and condensate molar fractions during continuous expansions in cloud chambers are then calculated using the classical nucleation theory and different droplet growth laws. Comparison of the nucleation rates and the condensate molar fractions obtained by the asymptotic solution with numerical simulations for initial pressures ranging from low $\left(p_{0}^{\prime}=1\right.$ bar) to high $\left(p_{0}^{\prime}=50 \mathrm{bar}\right)$ pressures shows complete qualitative and reasonable quantitative agreement despite some notable deviations near the onset of condensation resulting from the use of second order approximation to the activation function of nucleation. Maximum nucleation rates obtained by the asymptotic and numerical solutions are within an order of magnitude (higher in the asymptotic solution).

This investigation shows that the shape of the nucleation curve, independent of the particular nucleation rate equation to be employed, can be determined satisfactorily both by numerical simulations and by the asymptotic solution (although there are some differences between the solutions). The approach toward saturated thermodynamic equilibrium is also achieved in both solutions. In cases where a very precise quantitative information of the nucleation curve is required, the numerical simulations seem to yield quite satisfactory results whereas the second order asymptotic theory would yield results within an order of magnitude compared to those of numerical simulations (higher approximations ofthe activation function near its turning point can definitely improve the results; however, the analytical nature of the solution will then be destroyed). Noting that nucleation theories and nucleation rate measurements are at least off within one or two orders of magnitude and taking into account 
theanalytical structure of the condensation zones exhibited by the second order asymptotic solution, the results of the second order asymptotic theory should also be considered as being satisfactory for any practical application.

\section{ACKNOWLEDGMENTS}

One of us (C.F.D.) acknowledges the support by the J. M. Burgers Center during his visit in Eindhoven. This research was supported in part by the Netherlands Foundation of Fundamental Research on Matter (FOM) under Grant No. ETN 00.2347, and in part by the Scientific and Technical Research Council of Turkey (TÜBITAK) and by the Turkish Academy of Sciences (TÜBA).

\section{APPENDIX A: ASYMPTOTIC SOLUTION OF THE CONDENSATION RATE EQUATION IN THE TRANSITION REGIME}

In the initial growth (IGZ) and further growth (FGZ) zones where $d B / d t=O(1)$, the asymptotic expression for $y$ and $d y / d t$ follow from the solution of the rate Eq. (53) by Laplace's method ${ }^{31,32}$ for an endpoint minimum at $\tau=t$ in the limit $K \rightarrow 0$ and $\lambda_{\mathrm{HK}}, \lambda_{C} \rightarrow \infty$ with $\lambda_{C} / \lambda_{\mathrm{HK}}=$ finite.

$$
\begin{aligned}
y(t)= & {\left[2 \lambda_{C} \Omega_{C}(t)\right]^{3 / 2} K^{5 / 2}\left(-\frac{d B}{d t}\right)^{-5 / 2} \frac{\Sigma(t)}{n(t)} } \\
& \times \exp \left[-K^{-1} B(t)\right]\left\{\frac{3}{4} \sqrt{\pi}\left[1+2 q^{2}(t)\right]\right. \\
& \times \exp \left[-K^{-1} \frac{d B}{d t} b(t)\right]+3 q(t)\left[1-K^{-1} \frac{d B}{d t} b(t)\right] \\
& \left.+q^{3}(t)\right\}
\end{aligned}
$$

and

$$
\begin{aligned}
\frac{d y}{d t}= & r_{\mathrm{cr}}^{3}(t) \frac{\Sigma(t)}{n(t)} \exp \left[-K^{-1} B(t)\right] \\
& +3\left[2 \lambda_{C} \Omega_{C}(t)\right]^{3 / 2} K^{3 / 2}\left(-\frac{d B}{d t}\right)^{-3 / 2} \frac{\Sigma(t)}{n(t)} \\
& \times \exp \left[-K^{-1} B(t)\right]\left\{\frac{\sqrt{\pi}}{4}\left[1+2 q^{2}(t)\right]\right. \\
& \left.\times \exp \left[-K^{-1} \frac{d B}{d t} b(t)\right]+q(t)\right\},
\end{aligned}
$$

where $a(t), b(t)$, and $q(t)$ are defined by

$$
\begin{aligned}
& a(t) \equiv r_{\mathrm{cr}}(t)-\frac{\lambda_{C} \Omega_{C}(t)}{\lambda_{\mathrm{HK}} \Omega_{\mathrm{HK}}(t)}, \\
& b(t) \equiv \frac{1}{2 \lambda_{C} \Omega_{C}(t)}\left[\frac{\lambda_{C} \Omega_{C}(t)}{\lambda_{\mathrm{HK}} \Omega_{\mathrm{HK}}(t)}\right]^{2}, \\
& q(t) \equiv a(t) \sqrt{\frac{(-d B / d t)}{2 K \lambda_{C} \Omega_{C}(t)}},
\end{aligned}
$$

and where $d B / d t$ is evaluated from

$$
\frac{d B}{d t}=\frac{\partial B}{\partial p} \frac{d p}{d t}+\frac{\partial B}{\partial T} \frac{d T}{d t}+\frac{\partial B}{\partial y} \frac{d y}{d t} .
$$

Equations (A1)-(A6) form a transcendental system for $y$ and $d y / d t$ in FGZ and can be solved iteratively starting with the local frozen solution (usually a single iteration suffices). In particular the expressions for $y$ and $d y / d t$ in IGZ can be simplified by considering the frozen limit of the thermodynamic functions, i.e., $B \rightarrow B_{f}, \quad \Sigma \rightarrow \Sigma_{f}, \quad r_{\mathrm{cr}} \rightarrow r_{\mathrm{cr}, f}$, $\Omega_{C} \rightarrow \Omega_{C, f}$, and $\Omega_{\mathrm{HK}} \rightarrow \Omega_{\mathrm{HK}, f}$ where by subscript $f$ we mean the frozen (isentropic) value of the function.

In the rapid growth zone (RGZ) and the embedded onset zone $(\mathrm{OZ}), d B / d t$ diminishes to $O\left(K^{1 / 2}\right)$ as $K \rightarrow 0$ by definition. From the solution of the rate Eq. (53) by Laplace's $\operatorname{method}^{15,16}$ for an end point minimum we obtain the following expressions for $y, d y / d t$, and $d^{2} y / d t^{2}$ in the second order approximation to the activation function $B(t)$

$$
\begin{aligned}
y(t)= & {\left[2 \lambda_{C} \Omega_{C}(t)\right]^{3 / 2}[2 \beta(t)]^{-5 / 4} \frac{\Sigma(t)}{n(t)} \exp \left[\frac{\gamma^{2}(t)}{8 \beta(t)}\right] \exp \left[-K^{-1} B(t)\right]\left(\left\{\frac{3}{4} \sqrt{\pi} D_{-5 / 2}\left[\frac{\gamma(t)-2 \beta(t) b(t)}{\sqrt{2 \beta(t)}}\right]\right.\right.} \\
& \left.+\frac{3}{2} \sqrt{\pi} \bar{q}^{2}(t) D_{-3 / 2}\left[\frac{\gamma(t)-2 \beta(t) b(t)}{\sqrt{2 \beta(t)}}\right]\right\} \exp \left[\frac{\gamma(t) b(t)-\beta(t) b^{2}(t)}{2}\right]+\left\{\bar{q}^{3}(t)+3 \bar{q}(t) b(t)[2 \beta(t)]^{1 / 2}\right\} \\
& \left.\times D_{-1}\left[\frac{\gamma(t)}{\sqrt{2 \beta(t)}}\right]+3 \bar{q}(t) D_{-2}\left[\frac{\gamma(t)}{\sqrt{2 \beta(t)}}\right]\right),
\end{aligned}
$$




$$
\begin{aligned}
\frac{d y}{d t}= & r_{\mathrm{cr}}^{3}(t) \frac{\Sigma(t)}{n(t)} \exp \left[-K^{-1} B(t)\right]+3\left[2 \lambda_{C} \Omega_{C}(t)\right]^{3 / 2}[2 \beta(t)]^{-3 / 4} \frac{\Sigma(t)}{n(t)} \exp \left[\frac{\gamma^{2}(t)}{8 \beta(t)}\right] \exp \left[-K^{-1} B(t)\right] \\
& \times\left(\frac{\sqrt{\pi}}{4}\left\{D_{-3 / 2}\left[\frac{\gamma(t)-2 \beta(t) b(t)}{\sqrt{2 \beta(t)}}\right]+2 \bar{q}^{2}(t) D_{-1 / 2}\left[\frac{\gamma(t)-2 \beta(t) b(t)}{\sqrt{2 \beta(t)}}\right]\right\} \exp \left[\frac{\gamma(t) b(t)-\beta(t) b^{2}(t)}{2}\right]\right. \\
& \left.+\bar{q}(t) D_{-1}\left[\frac{\gamma(t)}{\sqrt{2 \beta(t)}}\right]\right),
\end{aligned}
$$

and

$$
\begin{aligned}
\frac{d^{2} y}{d t^{2}}= & \frac{1}{\Omega_{C}(t)} \frac{d \Omega_{C}}{d t} \frac{d y}{d t}+r_{\mathrm{cr}}^{3}(t) \frac{\Sigma(t)}{n(t)} \exp \left[-K^{-1} B(t)\right]\left[\frac{3}{r_{\mathrm{cr}}(t)} \frac{d r_{\mathrm{cr}}}{d t}+\frac{1}{\Sigma(t)} \frac{d \Sigma}{d t}-\frac{1}{n(t)} \frac{d n}{d t}-\frac{1}{\Omega_{C}(t)} \frac{d \Omega_{C}}{d t}+\gamma(t)\right] \\
& +3 \lambda_{\mathrm{HK}} \Omega_{\mathrm{HK}}(t) r_{\mathrm{cr}}^{2}(t) \frac{\Sigma(t)}{n(t)} \exp \left[-K^{-1} B(t)\right]+\frac{3}{4} \sqrt{\pi}\left[2 \lambda_{C} \Omega_{C}(t)\right]^{3 / 2}[2 \beta(t)]^{-1 / 4} \frac{\Sigma(t)}{n(t)} \\
& \times \exp \left[-K^{-1} B(t)\right] \exp \left[\frac{\gamma(t) b(t)-\beta(t) b^{2}(t)}{2}\right] \exp \left[\frac{\gamma^{2}(t)}{8 \beta(t)}\right] D_{-1 / 2}\left[\frac{\gamma(t)-2 \beta(t) b(t)}{\sqrt{2 \beta(t)}}\right],
\end{aligned}
$$

where $D_{\nu}(x)$ is Whittaker's parabolic cylinder function ${ }^{34,35}$ of $x$ and of order $\nu$ and where

$$
\begin{aligned}
& \bar{q}(t) \equiv a(t) \sqrt{\frac{\sqrt{2 \beta(t)}}{2 \lambda_{C} \Omega_{C}(t)},} \\
& \gamma(t) \equiv-K^{-1} \frac{d B}{d t}>0, \\
& \beta(t) \equiv \frac{1}{2} K^{-1} \frac{d^{2} B}{d t^{2}}>0,
\end{aligned}
$$

with $d B / d t$ evaluated from Eq. (A6) and $d^{2} B / d t^{2}$ evaluated by

$$
\begin{aligned}
\frac{d^{2} B}{d t^{2}}= & \frac{\partial B}{\partial p} \frac{d^{2} p}{d t^{2}}+\frac{\partial B}{\partial T} \frac{d^{2} T}{d t^{2}}+\frac{\partial B}{\partial y} \frac{d^{2} y}{d t^{2}}+\frac{\partial^{2} B}{\partial p^{2}}\left(\frac{d p}{d t}\right)^{2} \\
& +\frac{\partial^{2} B}{\partial T^{2}}\left(\frac{d T}{d t}\right)^{2}+\frac{\partial^{2} B}{\partial y^{2}}\left(\frac{d y}{d t}\right)^{2}+2\left(\frac{\partial^{2} B}{\partial p \partial T} \frac{d p}{d t} \frac{d T}{d t}\right. \\
& \left.+\frac{\partial^{2} B}{\partial p \partial y} \frac{d p}{d t} \frac{d y}{d t}+\frac{\partial^{2} B}{\partial T \partial y} \frac{d T}{d t} \frac{d y}{d t}\right) .
\end{aligned}
$$

Equations (A7)-(A13) together with the governing Eqs. (11)-(13) can be solved iteratively for the flow variables $n$, $T$, and $y$ in RGZ and $\mathrm{OZ}$ starting with a nearby upstream solution. In particular the solution for the flow field at the relative onset point follows in the limit as

$$
\gamma \rightarrow \gamma_{l} \equiv 0, \beta \rightarrow \beta_{l}, \Sigma \rightarrow \Sigma_{l}, \text { etc. }
$$

In the nucleation zone with growth (NZ) the asymptotic expressions for $y$ and $d y / d t$ follow from the solution of the rate Eq. (53) where the relative onset point $t_{l}$ acts as an interior minimum for Laplace's method. Consequently we obtain

$$
\begin{aligned}
y(\psi)= & \nu_{l}\left(\frac { 3 } { 4 } \sqrt { \pi } \left\{D_{-5 / 2}\left[-\sqrt{2}\left(\beta_{l}^{1 / 2} b_{l}+\psi\right)\right]\right.\right. \\
& \left.+2 \bar{q}_{l}^{2} D_{-3 / 2}\left[-\sqrt{2}\left(\beta_{l}^{1 / 2} b_{l}+\psi\right)\right]\right\} \\
& \times \exp \left[-\frac{\left(\beta_{l}^{1 / 2} b_{l}+\psi\right)^{2}}{2}\right]+\left[\sqrt{\frac{\pi}{2}} \bar{q}_{l}^{3}\right. \\
& \left.+3 \sqrt{\pi} \bar{q}_{l}\left(\beta_{l}^{1 / 2} b_{l}+\psi\right)\right](1+\operatorname{erf} \psi) \\
& \left.+3 \bar{q}_{l} \exp \left(-\psi^{2}\right)\right),
\end{aligned}
$$

$$
\begin{aligned}
\frac{d y}{d t}= & r_{\mathrm{cr}}^{3}(t) \frac{\sum(t)}{n(t)} \exp \left[-K^{-1} B(t)\right] \\
& +\frac{3}{4} \sqrt{\pi} \nu_{l}\left(2 \beta_{l}\right)^{1 / 2}\left(\left\{D_{-3 / 2}\left[-\sqrt{2}\left(\beta_{l}^{1 / 2} b_{l}+\psi\right)\right]\right.\right. \\
& \left.+2 \bar{q}_{l}^{2} D_{-1 / 2}\left[-\sqrt{2}\left(\beta_{l}^{1 / 2} b_{l}+\psi\right)\right]\right\} \\
& \left.\times \exp \left[-\frac{\left(\beta_{l}^{1 / 2} b_{l}+\psi\right)^{2}}{2}\right]+2 \sqrt{2} \bar{q}_{l}(1+\operatorname{erf} \psi)\right),
\end{aligned}
$$

where

$$
\begin{aligned}
& \psi \equiv \sqrt{\beta_{l}}\left(t-t_{l}\right) \geqslant 0, \\
& \nu_{l} \equiv\left[2 \lambda_{C} \Omega_{C l}\right]^{3 / 2}\left(2 \beta_{l}\right)^{-5 / 4} \frac{\Sigma_{l}}{n_{l}} \exp \left[-K^{-1} B_{l}\right],
\end{aligned}
$$

with subscript $l$ denoting values of functions evaluated at $t=t_{l}$ [e.g., $\Omega_{C l}=\Omega_{C}\left(t_{l}\right)$, etc.]. In particular we recover the solution at $t=t_{l}$ in the limit as $\psi \rightarrow 0$. When $\psi \gg 1$, we get

$$
\begin{aligned}
y(\psi) \sim & \sqrt{\pi} \nu_{l}\left[2^{5 / 4}\left(\beta_{l}^{1 / 2} b_{l}+\psi\right)^{3 / 2}+6 \bar{q}_{l}\left(\beta_{l}^{1 / 2} b_{l}+\psi\right)\right. \\
& \left.+(3) 2^{3 / 4} \bar{q}_{l}^{2}\left(\beta_{l}^{1 / 2} b_{l}+\psi\right)^{1 / 2}+\sqrt{2} \bar{q}_{l}^{3}\right] .
\end{aligned}
$$


The growth law given by Eq. (A19) can not persist in the droplet growth zone (DGZ) where thermodynamic equilibrium is established. Using once more Laplace's method for an interior minimum, the solution of the rate Eq. (53) in this zone can be cast into the form

$$
\begin{aligned}
& y=\left[\sqrt{\bar{R}}+\left(\sqrt{2 \pi} \nu_{l}\right)^{1 / 3} \bar{q}_{l}\right]^{3}, \\
& \frac{d y}{d t}=\frac{3}{2} \frac{\Omega_{C}(t)}{\Omega_{C l}}\left(\sqrt{2 \pi} \nu_{l}\right)^{2 / 3}\left(2 \beta_{l}\right)^{1 / 2} \\
& \times \frac{\left[\sqrt{\bar{R}}+\left(\sqrt{2 \pi} \nu_{l}\right)^{1 / 3} \bar{q}_{l}\right]^{2}}{\sqrt{\bar{R}}},
\end{aligned}
$$

with $\bar{R}$ satisfying the scaled relaxation rate equation

$$
\frac{d \bar{R}}{d \chi}=\frac{\Omega_{C}}{\Omega_{C l}},
$$

where the scaling laws

$$
\begin{aligned}
& \bar{R} \equiv \Lambda \beta_{l}^{1 / 3}\left[b_{l}+\int_{t_{l}}^{t} \frac{\Omega_{C}(\tau)}{\Omega_{C l}} d \tau\right], \\
& \chi \equiv \Lambda \beta_{l}^{1 / 3}\left(t-t_{l}\right),
\end{aligned}
$$

have been introduced with the scaling parameter $\Lambda$ given by

$$
\Lambda \equiv\left[2 \sqrt{\pi} \nu_{l}\right]^{2 / 3}\left(2 \beta_{l}\right)^{1 / 6} .
$$

The nonlinear droplet relaxation Eq. (A22) is of the same form as nonequilibrium internal mode excitation or chemical reaction with flow and tends to saturated equilibrium states $\left(\Omega_{C} \rightarrow 0\right.$ as $\left.\chi \rightarrow \infty\right)$. It can be solved by quadrature starting with an initial value $\hat{R}$ obtained by matching smoothly the solution of the nucleation zone with growth (NZ) with the droplet growth zone (DGZ) solution at a conveniently chosen point $\hat{\chi}$.

\section{APPENDIX B: ASYMPTOTIC SOLUTION OF THE CONDENSATION RATE EQUATION IN THE CONTINUUM REGIME}

The condensation rate equation of the continuum regime given by Eq. (49) can be solved asymptotically in the double limit as $K \rightarrow 0$ and $\lambda_{C} \rightarrow \infty$. In particular in the initial growth (IGZ) and further growth (FGZ) zones the asymptotic expressions for $y$ and $d y / d t$ follow by Laplace's method ${ }^{31,32}$ for an end point minimum at $\tau=t$ in the double limit given above.

$$
\begin{aligned}
y(t)= & \frac{3}{4} \sqrt{\pi}\left[2 \lambda_{C} \Omega_{C}(t)\right]^{3 / 2} K^{5 / 2}\left(-\frac{d B}{d t}\right)^{-5 / 2} \frac{\Sigma(t)}{n(t)} \\
& \times \exp \left[-K^{-1} B(t)\right] \exp \left[-K^{-1} \frac{d B}{d t} c(t)\right]
\end{aligned}
$$

$$
\begin{aligned}
\frac{d y}{d t}= & r_{\mathrm{cr}}^{3}(t) \frac{\sum(t)}{n(t)} \exp \left[-K^{-1} B(t)\right] \\
& +\frac{3}{4} \sqrt{\pi}\left[2 \lambda_{C} \Omega_{C}(t)\right]^{3 / 2} K^{3 / 2}\left(-\frac{d B}{d t}\right)^{-3 / 2} \frac{\sum(t)}{n(t)} \\
& \times \exp \left[-K^{-1} B(t)\right] \exp \left[-K^{-1} \frac{d B}{d t} c(t)\right],
\end{aligned}
$$

where $c(t)$ is defined by

$$
c(t) \equiv \frac{r_{\mathrm{cr}}^{2}(t)}{2 \lambda_{C} \Omega_{C}(t)}
$$

together with $d B / d t$ given by

$$
\frac{d B}{d t}=\frac{\partial B}{\partial p} \frac{d p}{d t}+\frac{\partial B}{\partial T} \frac{d T}{d t}+\frac{\partial B}{\partial y} \frac{d y}{d t} .
$$

Equations (B1)-(B4) together with Eqs. (11)-(13) can be solved iteratively for the flow field in IGZ and DGZ starting with the local frozen solution $(y \equiv 0)$. In particular the expressions in the initial growth zone (IGZ) can be simplified by considering the frozen limit for the thermodynamic functions, namely,

$$
T \rightarrow T_{f}, \quad n \rightarrow n_{f}, \quad B \rightarrow B_{f}, \quad \Sigma \rightarrow \Sigma_{f}, \quad \Omega_{C} \rightarrow \Omega_{C f} \text {, etc. }
$$

In the rapid growth zone (RGZ) and the embedded onset zone (OZ), where $d B / d t=O\left(K^{1 / 2}\right)$ as $K \rightarrow 0$, the asymptotic expressions for the condensate molar fraction $y$ and its derivatives $d y / d t$ and $d^{2} y / d t^{2}$ are obtained by Laplace's method $^{31,32}$ for an end point minimum taking into account the second order approximation of the activation function $B$.

$$
\begin{aligned}
y(t)= & \frac{3}{4} \sqrt{\pi}\left[2 \lambda_{C} \Omega_{C}(t)\right]^{3 / 2}[2 \beta(t)]^{-5 / 4} \frac{\Sigma(t)}{n(t)} \\
& \times \exp \left[-K^{-1} B(t)\right] \exp \left[\frac{\gamma^{2}(t)}{8 \beta(t)}\right] \\
& \times \exp \left[\frac{\gamma(t) c(t)-\beta(t) c^{2}(t)}{2}\right] \\
& \times D_{-5 / 2}\left[\frac{\gamma(t)-2 \beta(t) c(t)}{\sqrt{2 \beta(t)}}\right],
\end{aligned}
$$

$$
\begin{aligned}
\frac{d y}{d t}= & r_{\mathrm{cr}}^{3}(t) \frac{\Sigma(t)}{n(t)} \exp \left[-K^{-1} B(t)\right] \\
& +\frac{3}{4} \sqrt{\pi}\left[2 \lambda_{C} \Omega_{C}(t)\right]^{3 / 2}[2 \beta(t)]^{-3 / 4} \frac{\Sigma(t)}{n(t)} \\
& \times \exp \left[-K^{-1} B(t)\right] \exp \left[\frac{\gamma^{2}(t)}{8 \beta(t)}\right] \\
& \times \exp \left[\frac{\gamma(t) c(t)-\beta(t) c^{2}(t)}{2}\right] \\
& \times D_{-3 / 2}\left[\frac{\gamma(t)-2 \beta(t) c(t)}{\sqrt{2 \beta(t)}}\right],
\end{aligned}
$$

and

and 


$$
\begin{aligned}
\frac{d^{2} y}{d t^{2}}= & \frac{1}{\Omega_{C}(t)} \frac{d \Omega_{C}}{d t} \frac{d y}{d t}+r_{\mathrm{cr}}^{3}(t) \frac{\Sigma(t)}{n(t)} \exp \left[-K^{-1} B(t)\right]\left[\frac{3}{r_{\mathrm{cr}}(t)} \frac{d r_{\mathrm{cr}}}{d t}+\frac{1}{\Sigma(t)} \frac{d \Sigma}{d t}-\frac{1}{n(t)} \frac{d n}{d t}-\frac{1}{\Omega_{C}(t)} \frac{d \Omega_{C}}{d t}+\gamma(t)\right] \\
& +3 \lambda_{C} \Omega_{C}(t) r_{\mathrm{cr}}(t) \frac{\Sigma(t)}{n(t)} \exp \left[-K^{-1} B(t)\right]+\frac{3}{4} \sqrt{\pi}\left[2 \lambda_{C} \Omega_{C}(t)\right]^{3 / 2}[2 \beta(t)]^{-1 / 4} \frac{\Sigma(t)}{n(t)} \exp \left[-K^{-1} B(t)\right] \exp \left[\frac{\gamma^{2}(t)}{8 \beta(t)}\right] \\
& \times \exp \left[\frac{\gamma(t) c(t)-\beta(t) c^{2}(t)}{2}\right] D_{-1 / 2}\left[\frac{\gamma(t)-2 \beta(t) c(t)}{\sqrt{2 \beta(t)}}\right],
\end{aligned}
$$

where $D_{\nu}(x)$ is Whittaker's parabolic cylinder function ${ }^{34,35}$ of $x$ and of order $\nu$ and where

$$
\begin{aligned}
& \gamma(t) \equiv-K^{-1} \frac{d B}{d t}>0, \\
& \beta(t) \equiv \frac{1}{2} K^{-1} \frac{d^{2} B}{d t^{2}}>0,
\end{aligned}
$$

with $d B / d t$ evaluated from Eq. (B4) and $d^{2} B / d t^{2}$ evaluated by

$$
\begin{aligned}
\frac{d^{2} B}{d t^{2}}= & \frac{\partial B}{\partial p} \frac{d^{2} p}{d t^{2}}+\frac{\partial B}{\partial T} \frac{d^{2} T}{d t^{2}}+\frac{\partial B}{\partial y} \frac{d^{2} y}{d t^{2}}+\frac{\partial^{2} B}{\partial p^{2}}\left(\frac{d p}{d t}\right)^{2} \\
& +\frac{\partial^{2} B}{\partial T^{2}}\left(\frac{d T}{d t}\right)^{2}+\frac{\partial^{2} B}{\partial y^{2}}\left(\frac{d y}{d t}\right)^{2}+2\left(\frac{\partial^{2} B}{\partial p \partial T} \frac{d p}{d t} \frac{d T}{d t}\right. \\
& \left.+\frac{\partial^{2} B}{\partial p \partial y} \frac{d p}{d t} \frac{d y}{d t}+\frac{\partial^{2} B}{\partial T \partial y} \frac{d T}{d t} \frac{d y}{d t}\right) .
\end{aligned}
$$

In particular the above asymptotic expressions at the relative onset point $t=t_{l}$ can be obtained in the limit as

$$
\gamma \rightarrow \gamma_{l} \equiv 0, \beta \rightarrow \beta_{l}, \text { etc. }
$$

where by subscript $l$ we mean evaluated at $t=t_{l}$.

In the nucleation zone with growth (NZ), where both nucleation and droplet growth are important, the asymptotic expressions for $y$ and $d y / d t$ follow from the rate Eq. (49) by Laplace's method ${ }^{31,32}$ for an interior minimum

$$
\begin{aligned}
y(\psi)= & \frac{3}{4} \sqrt{\pi} \nu_{l} \exp \left[-\frac{\left(\beta_{l}^{1 / 2} c_{l}+\psi\right)^{2}}{2}\right] \\
& \times D_{-5 / 2}\left[-\sqrt{2}\left(\beta_{l}^{1 / 2} c_{l}+\psi\right)\right], \\
\frac{d y}{d t}= & r_{\mathrm{cr}}^{3}(t) \frac{\Sigma(t)}{n(t)} \exp \left[-K^{-1} B(t)\right]+\frac{3}{4} \sqrt{\pi} \nu_{l}\left(2 \beta_{l}\right)^{1 / 2} \\
& \times \exp \left[-\frac{\left(\beta_{l}^{1 / 2} c_{l}+\psi\right)^{2}}{2}\right] D_{-3 / 2}\left[-\sqrt{2}\left(\beta_{l}^{1 / 2} c_{l}+\psi\right)\right],
\end{aligned}
$$

where the stretched coordinate $\psi$ and the parameter $\nu_{l}$ are defined by

$$
\psi \equiv \sqrt{\beta_{l}}\left(t-t_{l}\right) \geqslant 0
$$

and

$$
\nu_{l} \equiv\left[2 \lambda_{C} \Omega_{C l}\right]^{3 / 2}\left(2 \beta_{l}\right)^{-5 / 4} \frac{\Sigma_{l}}{n_{l}} \exp \left[-K^{-1} B_{l}\right]
$$

In particular we recover the solution at $t=t_{l}$ in the limit as $\psi \rightarrow 0$. Further downstream of the nucleation zone (NZ) where $\psi \gg 1$, we get

$$
y(\psi) \sim 2^{5 / 4} \sqrt{\pi} \nu_{l}\left(\beta_{l}^{1 / 2} c_{l}+\psi\right)^{3 / 2} .
$$

This limiting behavior of $y$ cannot persist in the droplet growth zone (DGZ) where saturated thermodynamic states are reached. In this case a different scaling law for the stretched coordinate should be used. Using Laplace's method for an interior minimum, one can easily show that the expressions for $y$ and $d y / d t$ in this case take the form

$$
y=[\bar{R}]^{3 / 2}
$$

and

$$
\frac{d y}{d t}=\frac{3}{2} \frac{\Omega_{C}(t)}{\Omega_{C l}}\left(\sqrt{2 \pi} \nu_{l}\right)^{2 / 3}\left(2 \beta_{l}\right)^{1 / 2} \sqrt{\bar{R}},
$$

where $\bar{R}$ satisfies the scaled relaxation rate equation

$$
\frac{d \bar{R}}{d \chi}=\frac{\Omega_{C}}{\Omega_{C l}}
$$

with the scaling laws introduced by

$$
\begin{aligned}
& \bar{R} \equiv \Lambda \beta_{l}^{1 / 3}\left[c_{l}+\int_{t_{l}}^{t} \frac{\Omega_{C}(\tau)}{\Omega_{C l}} d \tau\right], \\
& \chi \equiv \Lambda \beta_{l}^{1 / 3}\left(t-t_{l}\right) .
\end{aligned}
$$

The scaling parameter $\Lambda$ in the scaling laws (B21) and (B22) is given by

$$
\Lambda \equiv\left[2 \sqrt{\pi} \nu_{l}\right]^{2 / 3}\left(2 \beta_{l}\right)^{1 / 6} .
$$

The relaxation rate Eq. (B20) can be solved by quadrature starting with an initial value $\hat{R}$ obtained by matching smoothly the solution of the nucleation zone with growth (NZ) with the droplet growth zone (DGZ) solution at some point $\hat{\chi}$. It is also worthwhile to mention that a simplified version of the asymptotic solution of the rate equation in the continuum regime appeared first in the M.S. thesis by Verschueren. ${ }^{36}$ 


\section{APPENDIX C: THERMODYNAMIC DATA AND NORMALIZATION OF NUCLEATION AND DROPLET GROWTH THEORIES}

We herein exhibit the thermodynamic properties that enter the asymptotic theory for the condensation of water vapor in nitrogen. The molar latent heat of condensation $L^{\prime}\left(T^{\prime}\right)$ for water in the range of temperatures investigated is given by the linear relation

$$
L^{\prime}\left(T^{\prime}\right)=5.6857 \times 10^{4}-42.75 T^{\prime} \quad(\mathrm{J} / \mathrm{mol})
$$

in agreement with Eq. (10), where $T^{\prime}$ is measured in Kelvin. The planar saturation pressure $p_{v s, \infty}^{\prime}$ at $T^{\prime}$ is then evaluated by the Clausius-Clapeyron relation and is given by

$$
\begin{aligned}
p_{v s, \infty}^{\prime}\left(T^{\prime}\right)= & 610.8 \exp \left[-\frac{42.75}{\mathscr{R}} \ln \frac{T^{\prime}}{273.15}\right. \\
& \left.-\frac{5.6857 \times 10^{4}}{\mathscr{B}}\left(\frac{1}{T^{\prime}}-\frac{1}{273.15}\right)\right]
\end{aligned}
$$

where $\mathscr{B}=8.31441 \mathrm{~J} / \mathrm{mol} \mathrm{K}$ is the universal gas constant. The density of liquid water is taken at a constant value of $\rho_{l}^{\prime}=995 \mathrm{~kg} / \mathrm{m}^{3}$. the molar specific heats at constant pressure of water vapor and nitrogen are, respectively, given by $c_{p v}^{\prime}$ $=c_{p \mathrm{H}_{2} \mathrm{O}}^{\prime}=32.6693 \mathrm{~J} / \mathrm{mol} \mathrm{Kand} c_{p i}^{\prime}=c_{p \mathrm{~N}_{2}}^{\prime}=29.1615 \mathrm{~J} / \mathrm{mol} \mathrm{K}$. The variation of the surface tension $\sigma^{\prime}$ with temperature is taken to be of the form

$$
\sigma^{\prime}\left(T^{\prime}\right)=0.111773\left(1-\frac{T^{\prime}}{647.3}\right)^{0.712021}(\mathrm{~N} / \mathrm{m})
$$

and the diffusion coefficient $D^{\prime}$ is evaluated from

$$
D^{\prime}=\frac{2.45}{p^{\prime}}\left(\frac{T^{\prime}}{295}\right)^{2.085}\left(\mathrm{~m}^{2} / \mathrm{s}\right),
$$

where $T^{\prime}$ is measured in Kelvin and $p^{\prime}$ in pascal.

With the above thermodynamic properties we can normalize the nucleation rate equation and the continuum and Hertz-Knudsen droplet growth laws and identify the thermodynamic functions and parameters that enter the asymptotic theory. For the nucleation rate equation we use the classical theory given by

$$
J^{\prime}=\sqrt{\frac{2}{\pi} \sigma^{\prime}\left(T^{\prime}\right)}\left(m_{v}^{\prime}\right)^{-3 / 2} \frac{\rho_{v}^{\prime 2}}{\rho_{l}^{\prime}} \exp \left(-\frac{\Delta G^{*}}{k T^{\prime}}\right),
$$

where $m_{v}^{\prime}$ and $\rho_{v}^{\prime}$ are, respectively, the mass of a single vapor molecule and the mass density of the vapor and $\Delta G^{*} /\left(k T^{\prime}\right)$ is Gibbs formation energy given by

$$
\frac{\Delta G^{*}}{k T^{\prime}}=\frac{16}{3} \pi\left(\frac{m_{v}^{\prime}}{\rho_{l}^{\prime} \ln S}\right)^{2}\left[\frac{\sigma^{\prime}\left(T^{\prime}\right)}{k T^{\prime}}\right]^{3}
$$

with $S$ denoting the supersaturation ratio defined by $S$ $\equiv p_{v}^{\prime} / p_{v s, \infty}^{\prime}\left(T^{\prime}\right)$. Using the expression for the surface tension given by Eq. (C3) and the expression for the saturation pressure of water vapor given by Eq. (C2) and relating $\rho_{v}^{\prime}$ to the normalized thermodynamic coordinates $(p, T, y)$, Eq. (C5) can be cast into the normalized form

$$
J \equiv \frac{J^{\prime}}{\zeta^{\prime}}=\Sigma(p, T, y) \exp \left[-K^{-1} B(p, T, y)\right]
$$

in such a way that $\Sigma=O(1)$ and $B=O(1)$ during the period of significant nucleation rates where the normalization constant of nucleation $\zeta^{\prime}$ is given by

$$
\zeta^{\prime}=0.26675\left(m_{v}^{\prime}\right)^{-3 / 2} y_{v o}^{2} \frac{\left(\mu_{v} n_{s}^{\prime}\right)^{2}}{\rho_{l}^{\prime}},
$$

the nucleation parameter $K$ is given by

$$
K=42.7406\left(\frac{\rho_{l}^{\prime}}{m_{v}^{\prime}}\right)^{2}\left(k T_{s}^{\prime}\right)^{3} .
$$

The pre-exponential function $\Sigma$ and the activation function $B$ assume the form

$$
B(p, T, y)=[f(T)]^{3}[\ln S(p, T, y)]^{-2}
$$

and

$$
\Sigma(p, T, y)=\sqrt{\frac{f(T)}{T^{3}}}\left(\frac{1-y / y_{v o}}{1-y}\right)^{2} p^{2}
$$

with the normalized surface tension $f(T)$ and the supersaturation $S(p, T, y)$ given by

$$
f(T)=\frac{1}{T}\left[1-\frac{T_{s}^{\prime}}{647.3} T\right]^{0.712021}
$$

and

$$
\begin{aligned}
S(p, T, y) \equiv & \frac{p_{v}^{\prime}}{p_{v s, \infty}^{\prime}\left(T^{\prime}\right)} \\
= & \frac{p_{s}^{\prime}}{610.8}\left(\frac{y_{v o}-y}{1-y}\right) p \exp \left[A_{1} \ln \left(\frac{T_{s}^{\prime}}{273.15} T\right)\right. \\
& \left.+\frac{B_{1}}{T_{s}^{\prime}}\left(\frac{1}{T}-\frac{T_{s}^{\prime}}{273.15}\right)\right],
\end{aligned}
$$

where $A_{1}=5.141$ and $B_{1}=6.838 \times 10^{3}$. The critical radius $\left(r_{\mathrm{cr}}^{\prime}\right)$ is defined by the Gibbs-Thomson relation

$$
r_{\mathrm{cr}}^{\prime}=\frac{2 m_{v}^{\prime}}{\rho_{l}^{\prime}} \frac{\sigma^{\prime}}{k T^{\prime}} \frac{1}{\ln S}
$$

which in normalized form takes the form

$$
r_{\mathrm{cr}}(p, T, y) \equiv \frac{r_{\mathrm{cr}}^{\prime}}{r_{d}^{\prime}}=\frac{0.22355 m_{v}^{\prime}}{r_{d}^{\prime} \rho_{l}^{\prime} k T_{s}^{\prime}} \frac{f(T)}{\ln S(p, T, y)} .
$$

The Hertz-Knudsen droplet growth law given by Eqs. (16) and (25) (neglecting the Kelvin effect and taking the mass accommodation coefficient $\alpha_{M}^{\prime}$ equal to unity) assumes the normalized form

$$
\frac{d r}{d t}=\lambda_{\mathrm{HK}} \Omega_{\mathrm{HK}}(p, T, y),
$$

where the Hertz-Knudsen growth parameter $\lambda_{\mathrm{HK}}$ is given by

$$
\lambda_{\mathrm{HK}}=\frac{6108.0 t_{r}^{\prime}}{r_{d}^{\prime} \rho_{l}^{\prime} \sqrt{2 \pi T_{s}^{\prime} \mathscr{R} / \mu_{v}}}\left(\frac{273.15}{T_{s}^{\prime}}\right)^{A_{1}}
$$


and the Hertz-Knudsen growth function $\Omega_{\mathrm{HK}}$ (of order unity) is identified as

$$
\begin{aligned}
\Omega_{\mathrm{HK}}(p, T, y)= & \frac{1}{10 T^{\left(A_{1}+0.5\right)}} \exp \left[-B_{1}\left(\frac{1}{T_{s}^{\prime} T}-\frac{1}{273.15}\right)\right] \\
& \times[S(p, T, y)-1] .
\end{aligned}
$$

Similarly the continuum growth law given by Eqs. (15)-(18) and (24) and which, in neglecting the Kelvin effect, assumes the form

$$
\frac{d r^{\prime}}{d t^{\prime}}=\frac{1}{r^{\prime}} \frac{D^{\prime} p_{v s, \infty}^{\prime}\left(T^{\prime}\right)}{\rho_{l}^{\prime}\left(1-y_{v o}\right) R_{v} T^{\prime}}(S-1)
$$

can be cast into the normalized form of Eq. (28), namely

$$
\frac{d r}{d t}=\frac{1}{r} \lambda_{C} \Omega_{C}(p, T, y),
$$

where the continuum growth parameter $\lambda_{C}$ and the continuum growth function $\Omega_{C}$ are identified as

$$
\lambda_{C}=\frac{1.4965 \times 10^{4} t_{r}^{\prime}}{\rho_{l}^{\prime}\left(1-y_{v o}\right) R_{v} T_{s}^{\prime} p_{s}^{\prime} r_{d}^{\prime 2}}\left(\frac{T_{s}^{\prime}}{295}\right)^{2.085}\left(\frac{273.15}{T_{s}^{\prime}}\right)^{A_{1}}
$$

and

$$
\begin{aligned}
\Omega_{C}(p, T, y)= & \frac{1}{10 p T^{\left(A_{1}-1.085\right)}} \exp \left[-B_{1}\left(\frac{1}{T_{s}^{\prime} T}-\frac{1}{273.15}\right)\right] \\
& \times[S(p, T, y)-1] .
\end{aligned}
$$

${ }^{1}$ J. Aitken, Trans. R. Soc. Edinburgh 30, 337 (1880/81).

${ }^{2}$ A. Stodola, Dampf-und Gasturbinen (Springer, Berlin, 1922).

${ }^{3}$ C. T. R. Wilson, Philos. Trans. R. Soc. London, Ser. A 189, 265 (1897).

${ }^{4}$ K. Oswatitsch, ZAMM 22, 1 (1942).

${ }^{5}$ P. P. Wegener and L. M. Mack, Adv. Appl. Mech. 5, 307 (1958).
${ }^{6}$ Nucleation, edited by A. C. Zettlemoyer (Dekker, New York, 1969).

${ }^{7}$ F. F. Abraham, Homogeneous Nucleation Theory (Academic, New York, 1974).

${ }^{8}$ J. L. Katz, J. Chem. Phys. 52, 4733 (1970).

${ }^{9}$ P. E. Wagner and R. Strey, J. Chem. Phys. 80, 5266 (1984).

${ }^{10}$ R. Strey, P. E. Wagner, and T. Schmeling, J. Chem. Phys. 84, 2325 (1986)

${ }^{11}$ C. Hung, M. Krasnopoler, and J. L. Katz, J. Chem. Phys. 90, 1856 (1989); 92, 7722 (1990)

${ }^{12}$ F. Peters and B. Paikert, Exps. Fluids 7, 521 (1989).

${ }^{13}$ P. A. Blythe and C. J. Shih, J. Fluid Mech. 76, 593 (1976).

${ }^{14}$ J. H. Clarke and C. F. Delale, Phys. Fluids 29, 1389 (1986)

${ }^{15}$ C. F. Delale, G. H. Schnerr, and J. Zierep, Phys. Fluids A 5, 2969 (1993).

${ }^{16}$ C. F. Delale, G. H. Schnerr, and J. Zierep, J. Fluid Mech. 287, 93 (1995).

${ }^{17}$ R. Becker and W. Döring, Ann. Phys. 24, 719 (1935).

${ }^{18}$ Y. B. Zeldovich, Acta Physicochim. USSR 18, 1 (1943).

${ }^{19}$ J. Lothe and G. M. Pound, J. Chem. Phys. 36, 2080 (1962).

${ }^{20}$ H. Reiss, J. L. Katz, and E. R. Cohen, J. Chem. Phys. 48, 5553 (1968).

${ }^{21}$ M. E. Fisher, Physics 3, 255 (1967).

${ }^{22}$ A. Dillmann and G. E. A. Meier, J. Chem. Phys. 94, 3872 (1991).

${ }^{23}$ C. F. Delale and G. E. A. Meier, J. Chem. Phys. 98, 9850 (1993).

${ }^{24}$ V. I. Kalikmanov and M. E. H. van Dongen, J. Chem. Phys. 103, 4250 (1995).

${ }^{25}$ G. Gyarmathy, Multiphase Science and Technology 1 (McGraw-Hill, New York, 1982), pp. 99-279.

${ }^{26}$ J. B. Young, Int. J. Heat Mass Transfer 34, 1649 (1991).

${ }^{27}$ J. B. Young, Int. J. Heat Mass Transfer 36, 2941 (1993).

${ }^{28}$ V. G. Chernyak and A. Ye. Margilevsky, Int. J. Heat Mass Transfer 32, 2127 (1989).

${ }^{29}$ R. E. Sampson and G. S. Springer, J. Fluid Mech. 36, 577 (1969); 40, 859 (1970).

${ }^{30}$ P. N. Shankar, J. Fluid Mech. 40, 395 (1970).

${ }^{31}$ A. Erdelyi, Asymptotic Expressions (Dover, New York, 1956).

${ }^{32}$ L. Sirovich, Techniques of Asymptotic Analysis (Springer, New York, 1971).

${ }^{33}$ M. J. E. H. Muitjens, Ph.D. dissertation, Eindhoven University of Technology, 1996.

${ }^{34}$ M. Abramowitz and A. Stegun, Handbook of Mathematical Functions (Dover, New York, 1965).

${ }^{35}$ I. S. Gradshteyn and I. M. Ryzhik, Tables of Integrals, Series and Products (Academic, New York, 1980).

${ }^{36}$ M. Verschueren, M.S. thesis, Eindhoven University of Technology, 1995. 